\title{
Kinematic waves in polar firn stratigraphy
}

\author{
Felix NG, ${ }^{1}$ Edward C. KING ${ }^{2}$ \\ ${ }^{1}$ Department of Geography, University of Sheffield, Winter Street, Sheffield S10 2TN, UK \\ E-mail: f.ng@sheffield.ac.uk \\ ${ }^{2}$ British Antarctic Survey, Natural Environment Research Council, Madingley Road, Cambridge CB3 OET, UK
}

\begin{abstract}
Radar studies of firn on the ice sheets have revealed complex folds on its internal layering that form from the interplay of snow accumulation and ice flow. A mathematical theory for these fold structures is presented, for the case where the radar cross section lies along the ice-flow direction and where the accumulation rate and ice-flow velocity are time-invariant. Our model, which accounts for firn densification, shows how 'information' (the depth and slope of isochrones) propagates on the radargram to govern its layer undulations. This leads us to discover universal rules behind the pattern of layer slopes on a distance-age domain and understand why the loci of layer-fold hinges curve, emerge and combine on the radargram to form closed loops that delineate areas of rising and plunging isochrones. We also develop a way of retrieving the accumulation rate distribution and layer ages from steady isochrone patterns. Analysis of a radargram from the onset zone of Bindschadler Ice Stream, West Antarctica, indicates that ice flow and accumulation rates have been steady there for the past $\sim \mathbf{4 0 0}$ years, and that spatial anomalies in the latter are coupled to surface topography induced by ice flow over the undulating ice-stream bed. The theory provides new concepts for the morphological interpretation of radargrams.
\end{abstract}

\section{INTRODUCTION}

Ground-based and airborne radar surveys are gradually revealing more of the internal structure of the Antarctic and Greenland ice sheets. Whether imaging firn or ice down to the bed, they show the widespread existence of layered reflections that can be traced over long distances, often hundreds of kilometres. 'Radar layers' interpreted as isochronal (of equal age) can extend information from ice cores laterally, and their geometry may be deciphered for ice-sheet history.

In the firn within the top $100 \mathrm{~m}$ or so below the surface, the internal stratigraphy is shaped by snow accumulation, firn compaction, and lateral advection by the underlying ice flow. Recent studies have linked the radar-detected layers here to density contrasts associated with thin layers of hoar or ice (Arcone and others, 2004) and shown that they are isochronal, making it possible to estimate the pattern of past accumulation rates from the depth of the layers, after their age and the firn density-depth profile have been established by firn-core analysis (e.g. Spikes and others, 2004; Anschütz and others, 2008). On a length scale of kilometres, the layers often undulate richly in character (e.g. Fig. 1a) due to the advective ice flow combined with non-uniform surface accumulation, the latter potentially reflecting influence by surface topography on the precipitation of snow and its redistribution by wind (King and others, 2004; Spikes and others, 2004; Frezzotti and others, 2007). The layer undulations thus record a spatially variable history of surface mass balance, which may be reconstructed by numerical inverse methods (Eisen, 2008), as has been done with isochrones deep in the ice (Nereson and others, 2000; Waddington and others, 2007; Koutnik, 2009).

As the radargram in Figure 1a shows, the pattern of isochrones in the firn can be extremely striking. Their undulations can look organized yet irregular, portraying fold-like structures that migrate with depth. Previous authors have reported these structures and simulated them numerically with forward models of layer-shape evolution (King and others, 2002; Arcone and others, 2005; Gray and others, 2005; Woodward and King, 2009), but these efforts have not gone beyond matching the observed patterns to derive comprehensive insights about their development. A fascinating hypothesis is that some fundamental mechanisms govern the folded layer architecture - however variable and complex it may seem. Here we explore this hypothesis by an analytical approach, in order to uncover the mechanisms and understand how the layer pattern encodes the system's forcings (ice-flow velocity and surface accumulation rate). The results should aid glaciologists seeking visual interpretation of radargrams.

In our theory below, we view the layer undulations as waveforms and use the terminology of geological folds to describe their configuration on the radargram. This is despite the fact that the undulations are not 'folds' in the geological sense of having been created by force-bearing deformation in the stratigraphy, but kinematic waves resulting from differential mass transport (Whitham, 1974). To keep things simple, we strip the problem to bare essentials and treat only two-dimensional (2-D) radar cross sections aligned with the ice flow. Steady forcings are assumed to enable insights into the mechanism of pattern formation. Accordingly, we emphasize that our theory is not meant for reconstructing time-variable forcings from layer patterns (the reader is referred to the papers by Waddington and others (2007) and Eisen (2008) for this important subject), although we will see that its use to interpret radargrams can reveal past unsteadiness. A key variable in our analysis is the slope of isochrones, which featured in an earlier theory (Parrenin and Hindmarsh, 2007) for the shape of deep radar layers in incompressible ice below the firn, down to the ice-sheet bed. While Parrenin and Hindmarsh's (2007) description shares common ground with ours, it addresses considerably more complex ice-flow fields than 


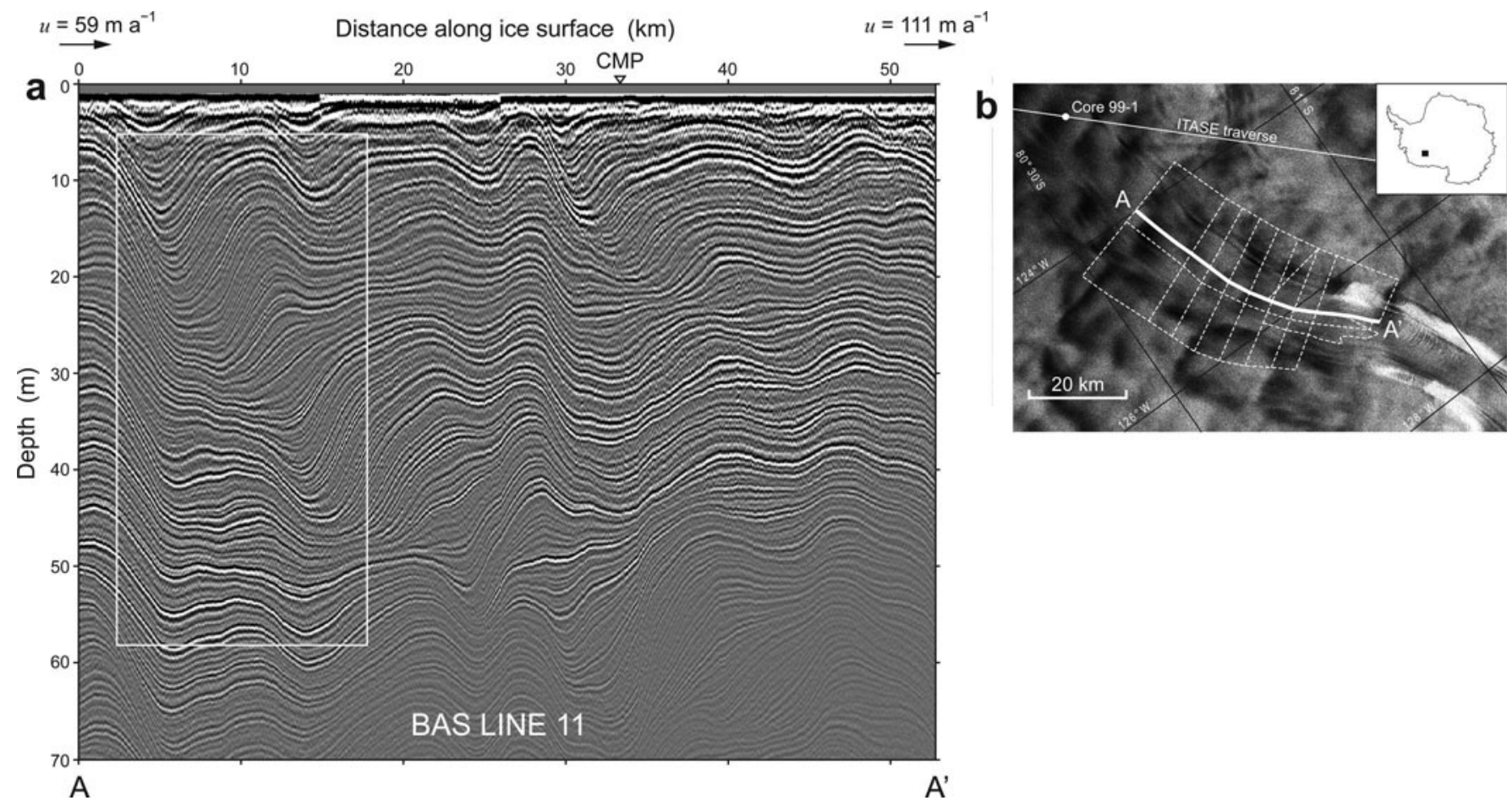

Fig. 1. (a) Radar cross section of the firn in the onset region of Bindschadler Ice Stream, West Antarctica, showing complex undulating stratigraphy. The vertical exaggeration is 600 times. The layered reflections are thought to result from interference of reflections from submetre-thick hoar or ice layers. The section is aligned roughly with the ice-flow direction (left to right). Surface velocities at its two ends from synthetic aperture radar (SAR) interferometry (Joughin and Tulaczyk, 2002), given at the top, indicate horizontal extension in the ice flow. CMP marks the common-midpoint survey that measured the wave speed in the firn column for converting the two-way travel time of radar traces to depth. No correction for surface elevation has been made, so the depth is referenced to the surface. The white box outlines the area studied in Figure 5. In Section 3.3 we use kinematic wave theory to analyse the isochrone pattern on this radargram. (b) Map of the study area, showing the ground traverse yielding the radargram in (a) (thick curve) on the SAR mosaic of the RADARSAT Antarctic Mapping Project, where bright areas delineate shear margins of the ice-stream tributary. Also shown is the network of radar traverses made during the same field season of 2001/02 austral summer (dashed curves), and Core 99-1 and the radar traverse of the International Trans-Antarctic Scientific Expedition (ITASE). Inset shows study area location in Antarctica.

ours where internal deformation and the effect of basal topography are significant. The flavour of our present study is also different because we account for firn densification and focus on the structural interpretation of radargrams based on their assembly of layer slopes, folds and fold hinges; both synthetic and real radargrams are examined below. Thus we provide the mathematical foundation for extending the work of Arcone and others (2005), who first seriously characterized layer folds as they appear on radargrams of firn where lateral ice flow is significant.

The outline of this paper is as follows. We give the analytic theory in Section 2, where we formulate equations for key mechanisms and consider what they predict for the layer morphology. This material is framed mainly with the 'forward' problem in mind: how forcings translate into patterns. In Section 3 we then turn to two case studies, where our focus shifts to the interpretation of radargrams and the 'inverse' problem of extracting the forcings from them. We propose a new inversion method based on subtracting the depth of isochrones, an approach that involves optimization but that differs from formal inverse methods (in which the forward model is optimized to fit observed layers; e.g. Eisen, 2008). In Section 3.3, our inversion method is applied to the radargram in Figure 1a, from Bindschadler Ice Stream, West Antarctica, and we discuss the glaciological implications of the results. Conclusions follow in Section 4.

\section{MATHEMATICAL THEORY}

\subsection{Formulation}

Consider a vertical cross section of firn, taken in the ice-flow direction, $x$. The firn moves in this direction at velocity $u(x, t)$ due to the underlying ice motion and receives surface accumulation at rate $a(x, t)$, where $t$ is time (see Fig. 2). Let $z(x, t)$ denote the depth of an isochronal layer or 'isochrone' below the surface. This coordinate ignores variations in surface elevation so that plotting $z$ against $x$ shows the isochrone under a flat horizon, as in Figure 1a. Our analysis of layer geometry considers also the slope of isochrones, the hinges occurring on them, the loci of these hinges (called hinge lines) and the migration velocity associated with hinge lines. Figure 2 introduces these terms, which are explained in more detail as the theory develops.

As each firn column translates, it receives surface accumulation but stretches or compresses laterally due to gradients of $u$. If we assume the rate of submergence at any depth to equal the surface burial rate a (expressed as a velocity), then a layer in the column deepens at the rate $\mathrm{d} z / \mathrm{d} t \quad(\equiv \partial z / \partial t+u \partial z / \partial x)=a-z \partial u / \partial x ;$ thus an isochrone evolves according to the partial differential equation

$$
\frac{\partial z}{\partial t}+\frac{\partial(u z)}{\partial x}=a
$$

This kinematic description assumes plane strain (zero flux in 
the $y$-direction and infinitely wide flow) and incompressibility for the firn. If necessary, the firn density can be used to convert the units of $a$ to $\mathrm{kg} \mathrm{m}^{-2} \mathrm{a}^{-1}$ or to m w.e. $\mathrm{a}^{-1}$.

Throughout this paper, $u$ and $a$ are taken as functions of $x$ only, not of time $t$. In the resulting steady flow, isochrones of different ages form a stationary pattern, in the sense that each isochrone deforms into the shape of successively older (and deeper) isochrones as it evolves in time. It is therefore possible to construct the entire pattern of isochrones by tracking the evolution of a single isochrone that lies initially at the surface. In this context, the variable $t$ takes the meaning of the age of the isochrones.

Equation (1) is simplistic because it ignores firn densification, which is clearly important in the depth range of interest. While this process does not fundamentally cause isochrones to undulate (we shall see a justification shortly), it affects their depth. Compaction of the firn under its own weight occurs at a rate dependent on rheology, temperature and accumulation rate, resulting in a firn density $\rho$ that increases nonlinearly with depth (e.g. Herron and Langway, 1980; Arthern and others, 2010). This causes material at depth to experience a submergence rate less than a. As the isochrones deepen, their shape retains a history of differential submergence caused by spatial variations in $a$, but densification reduces their mean separation.

In order to account for firn densification in tracking isochrones, several authors (Arcone and others, 2005; Eisen, 2008; Woodward and King, 2009) have posited a depthdependent submergence rate, scaled to the local accumulation rate by the density-depth profile $\rho(z)$, which is assumed invariant across the radargram. Specifically, Sorge's law (Bader, 1954) then gives the submergence rate as $\rho_{0} a / \rho(z)$, where $\rho_{0}$ is the firn surface density. Assuming steady forcings as before, we use these assumptions to formulate the mass-conservation equation

$$
\rho(z) \frac{\partial z}{\partial t}+\frac{\partial}{\partial x}\left(u(x) \int_{0}^{z} \rho(\zeta) \mathrm{d} \zeta\right)=\rho_{0} a(x),
$$

of which Equation (1) is now the special case $\rho(z) \equiv \rho_{0}$.

Although Equation (2) is a more complicated model of isochrone evolution than Equation (1), it can be put in the same form as the latter by the change of variable

$$
f(x, t)=\int_{0}^{z} \frac{\rho(\zeta)}{\rho_{0}} \mathrm{~d} \zeta
$$

which yields

$$
\frac{\partial f}{\partial t}+\frac{\partial(u f)}{\partial x}=a(x)
$$

The densification description adopted by us here (and by the aforementioned authors) thus merely introduces a depth correction that is uniform horizontally, implying that densification does not cause isochrones to undulate. However, in Section 4 we discuss caveats concerning this description that lead us to rethink its validity. The issues concern the assumption that $\rho$ is invariant with $x$, which affects not only Equation (2) but also the practice of compiling radargrams, where it is common to use wavespeed data from a few horizontal positions (obtained via common-midpoint surveys, as in Fig. 1a, or via $\rho$ in firn cores) to convert radar time traces to depth at all horizontal positions.

We proceed with Equation (4) and first show that it can be simplified to a canonical form for any spatially non-uniform
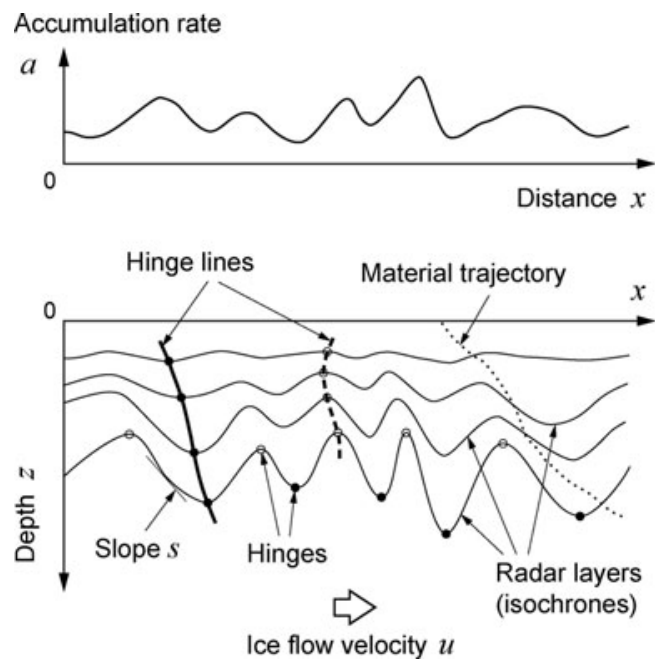

Fig. 2. Key terms and symbols in our mathematical model. Accumulation rate a (upper plot) and ice-flow velocity $u$ are external forcings generating the isochrone pattern (lower plot). An isochrone has depth profile $z(x)$ and local slope $s(=\partial z / \partial x)$, with $s$ defined to be positive and negative, respectively, where the layer plunges and rises along flow. Hinges are positions where $s=0$ and can be troughs or crests, as indicated by the solid dots and open circles on the deepest layer. The locus of hinges from different layers tracing the axes of a fold forms a hinge line. Examples of two types of hinge lines are shown: a trough line (bold curve) and a crest line (dashed curve). As we descend on a hinge line (with depth $z$ ), the hinge migrates in the sense that its horizontal position $x$ varies; the corresponding layer age $t$ increases. $\mathrm{d} x / \mathrm{d} t$ is the local hinge migration velocity, and $(\mathrm{d} x / \mathrm{d} z)^{-1}$ is the local dip of the hinge line. In our theory, Equations (20) and (23) predict these quantities.

flow velocity $u(x)>0$. Define the 'transformed depth' variable $Z(x, t)=u(x) f(x, t) / u_{0}$, where $\left.u_{0}=u(x)=0\right)$ is a constant reference velocity, here taken at the up-flow end of our domain. Then Equation (4) becomes

$$
\frac{\partial Z}{\partial t}+u(x) \frac{\partial Z}{\partial x}=\frac{u(x) a(x)}{u_{0}}
$$

and changing the variable $x$ to $X$ ('transformed distance') with

$$
x=\int_{0}^{x} \frac{u_{0}}{u(x)} \mathrm{d} x
$$

yields the canonical kinematic wave equation

$$
\frac{\partial Z}{\partial t}+u_{0} \frac{\partial Z}{\partial X}=A(X) \equiv \frac{u(X)}{u_{0}} a(X) .
$$

Some radar surveys have been made where $u$ increases along flow, for example in the onset zones of ice streams, where we may suppose a linear velocity model

$$
u(x)=u_{0}(1+k x) ;
$$

in this case $Z=(1+k x) f$ and Equation (6) leads to

$$
k X=\ln (1+k x)
$$

and

$$
A(X)=\mathrm{e}^{k X} \mathrm{a}(X) .
$$

In the rest of this paper, we analyse the problem in the transformed coordinates and use lower-case symbols for them (write $x$ for $X, z$ for $Z$ and $a$ for $A$ ) for convenience, with the understanding that reduction to the canonical form has been made and that we are referring to transformed layer 


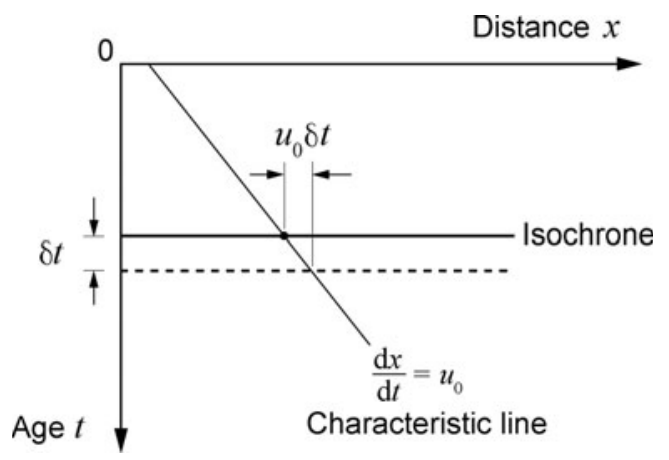

Fig. 3. The $x-t$ plane and the characteristic line (or 'characteristic') along which information travels. Depth $z$ and slope $s$ of isochrones, both functions of distance $x$ and age $t$, may be regarded as 3-D surfaces over the plane.

geometries unless we indicate otherwise. Then Equation (7) becomes

$$
\frac{\partial z}{\partial t}+u_{0} \frac{\partial z}{\partial x}=a(x)
$$

It is this simple wave equation that ultimately governs the spatial structure of the layer folds. Notice if $a(x)$ and $u_{0}$ are multiplied by the same factor, $t$ can always be rescaled to recover the original equation; this means that different forcings with the same ratio $a(x) / u_{0}$ generate the same isochrone pattern. Such non-uniqueness does not alter our findings on the pattern-formation mechanism below, but matters in inversions for the forcings from the layers (we shall see an example of such inversion in Section 3.3).

\subsection{Propagation of information}

Wave Equation (10) can be solved exactly by the method of characteristics (Carrier and Pearson, 1988) to give

$$
\frac{\mathrm{d} z}{\mathrm{~d} t}=a(x)
$$

along the characteristic line or 'characteristic'

$$
\frac{\mathrm{d} x}{\mathrm{~d} t}=u_{0} \text { or } x=x_{0}+u_{0} t
$$

Here $\mathrm{d} / \mathrm{d} t$ is the total derivative, and Equations (11) and (12) track the positions of material points $(x, z)$ parametrically. Material at position $x$ on an isochrone aged $t$ had the initial position $x_{0}$ at the surface when $t=0$. As it moves downglacier at velocity $u_{0}$, it deepens at a rate determined by the local accumulation rate (the use of transformed coordinates already accounts for the effects of densification and longitudinal flow extension/compression). Its $z$-value thus travels and varies along the characteristic. Figure 3 shows this idea on the $x-t$ plane (distance-age domain), where isochrones are lines of constant $t$. Note that in this paper, $t$ refers always to time or age, and not to the two-way travel time in the geophysical measurement of radar traces.

Now, integrating Equation (11) with $x$ taken from Equation (12) and with the initial condition $z=0$ at $t=0$, yields the explicit solution

$$
z(x, t)=\int_{0}^{t} a\left(x_{0}+u_{0} \tau\right) \mathrm{d} \tau=\frac{1}{u_{0}} \int_{x-u_{0} t}^{x} a(\zeta) \mathrm{d} \zeta
$$

(we have used the substitution $\zeta=x_{0}+u_{0} \tau$ ), which calculates each material particle's depth from the total overlying accumulation that it accrued as it travelled the horizontal

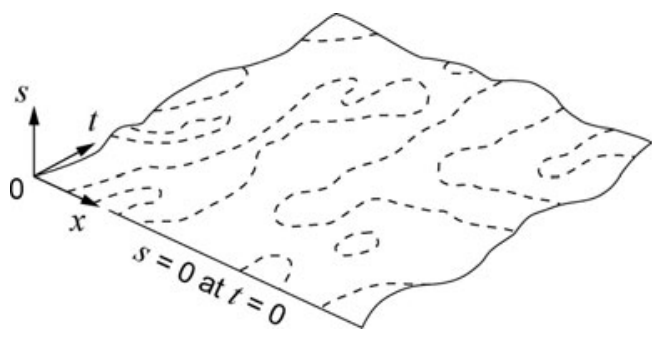

Fig. 4. The function $s(x, t)$ as a 3-D 'slope surface'. The axes are distance $x$, isochrone age $t$ and isochrone slope $s$. Intersection of the slope surface with the horizontal plane defines a system of hinge lines (dashed).

distance $u_{0} t$ to its current position. While $z$ evaluated with Equation (13) at a given time describes an isochrone's shape, $z(x, t)$ can be portrayed generally as a three-dimensional (3-D) surface over the $x-t$ plane. Isochrones on a radargram are projections of fixed-time samples of this surface onto the $x-z$ plane.

A key insight in this paper is that the slope of isochrones also obeys a kinematic wave equation. Let $s=\partial z / \partial x$ be the isochrone slope (Fig. 2). Since $z$ is referenced to the surface and $z$ and $x$ are transformed depth and distance, $s$ is not the true slope against the horizontal, but related to it. Differentiating Equation (10) with respect to $x$ gives

$$
\frac{\partial s}{\partial t}+u_{0} \frac{\partial s}{\partial x}=b(x)\left[\equiv \frac{\mathrm{d} a}{\mathrm{~d} x}\right]
$$

where $b$ is the spatial gradient of the surface accumulation rate. Both this wave equation and Equation (10) apply at positions $x$ where $a$ is differentiable (we assume this to be the case throughout our analysis), including where a changes smoothly from accumulation $(a>0)$ to ablation $(a<0)$. However, note that $a<0$ causes unconformity in the stratigraphy by removing parts of isochrones and making them discontinuous.

Solving Equation (14) with the method of characteristics yields

$$
\frac{\mathrm{d} s}{\mathrm{~d} t}=b(x) \text { on } x=x_{0}+u_{0} t,
$$

which describes how isochrone slope varies along the same characteristics (as in Equation (12)) in response to the overlying accumulation rate gradient. The corresponding integral solution satisfying $s=0$ at $t=0$ (the surface isochrone is ' $f$ lat') is

$$
\begin{gathered}
s(x, t)=\int_{0}^{t} b\left(x_{0}+u_{0} \tau\right) \mathrm{d} \tau=\frac{1}{u_{0}} \int_{x-u_{0} t}^{x} b(\zeta) \mathrm{d} \zeta \\
=\frac{1}{u_{0}}\left[a(x)-a\left(x-u_{0} t\right)\right] .
\end{gathered}
$$

Hence we may also regard $s(x, t)$ as a continuous surface over the $x$-t plane - we call this the 'slope surface' (Fig. 4) with fixed-time samples of it representing the slope variation of individual isochrones. And since slope information propagates on the $x-t$ plane, we may consider how $s$ varies across the radargram ( $x-z$ plane). These ideas help us understand the spatial structure of layer folding and are explored further in Section 2.4. The solutions above may in principle be found in the untransformed coordinates, but with much more algebra. 


\subsection{Retrieving surface accumulation rate}

Before turning to the folds, we use Equation (13) to establish two ways of extracting the accumulation rate pattern from isochrones that we build upon later. As with Equation (13), the following results rest on the steady-flow assumption and we are working in the transformed coordinates.

Method I is called 'shift-differencing'. Consider an isochrone aged $t$ and a slightly deeper, older isochrone aged $t+\delta t$. Shift these isochrones down-glacier and upglacier, respectively, by the distance $u_{0} \delta t / 2$, then subtract their depths. Equation (13) shows that

$$
\begin{aligned}
& z\left(x+0.5 u_{0} \delta t, t+\delta t\right)-z\left(x-0.5 u_{0} \delta t, t\right) \\
& =\frac{1}{u_{0}} \int_{x-0.5 u_{0} \delta t}^{x+0.5 u_{0} \delta t} a(\zeta) \mathrm{d} \zeta=a(x) \delta t+\mathrm{O}\left(\delta t^{3}\right) .
\end{aligned}
$$

Therefore, $a$ at position $x$ can be found by subtracting the depth of the younger isochrone at $x-u_{0} \delta t / 2$ from the depth of the older isochrone at $x+u_{0} \delta t / 2$, and dividing the result by $\delta \mathrm{tt}$. This method of estimating the accumulation rate pattern $a(x)$ avoids recasting Equation (4) as a formal inverse problem (e.g. Eisen, 2008) and has an error of order $\delta t^{2}$ caused by truncation of higher-order terms in Equation (17).

Method II involves subtracting the depths of the two isochrones without shifting them and also yields information about $a$. In this case

$$
\begin{gathered}
z(x, t+\delta t)-z(x, t)=\frac{1}{u_{0}} \int_{x-u_{0}(t+\delta t)}^{x-u_{0} t} a(\zeta) \mathrm{d} \zeta \\
=a\left(x-u_{0} t\right) \delta t+\mathrm{O}\left(\delta t^{2}\right) .
\end{gathered}
$$

The new differencing retrieves the accumulation pattern at a distance $u_{0} t$ up-glacier, with an error of order $\delta t$. If $t=0$, the upper isochrone is simply the firn surface, and Equation (18) shows that $a(x)$ can be inferred from a shallow isochrone (e.g. Gray and others, 2005; Woodward and King, 2009).

Both methods here rely on an isochrone separation small enough to limit truncation errors, but not so small that it becomes dominated by measurement uncertainty in the layer depths. Both methods require the age of isochrones (or at least their age difference) to be known, for example from a dated firn core at the site. As this is not always available, in Section 3.2 we develop a method that could be used without dated layers. Finally, while methods I and II will not work on isochrones that have been made discontinuous by ablation (or wherever $z$ is undefined), they can be used on continuous isochrones that bracket ablation unconformities, as long as the differentiability condition following Equation (14) is satisfied. In this case, shift-differencing the isochrones will make them intersect and retrieve negative as well as positive values of $a$.

\subsection{Structural architecture of layer undulations}

Recurrent properties of folded isochrones in the firn have been recognized in numerous flow-aligned radargrams from West Antarctica. It is noticed that the hinge axes of layer folds dip at angles that reflect a migration velocity less than the ice-flow velocity, and that these axes can dip up- as well as down-glacier (King and others, 2002; Arcone and others, 2005; Gray and others, 2005). By choosing suitable forms of $u(x)$ and $a(x)$, these authors have used numerical models of isochrone evolution to recreate layer patterns and simulate the observed dips. Arcone and others (2005), in particular, posed sinuisoidal and Gaussian functions for $a(x)$ to study the controls on the dip. Some radargrams also exhibit folds whose amplitudes increase with depth (Vaughan and others, 1999, 2004; King and others, 2002; Arcone and others, 2005; Gray and others, 2005), a property that has been observed on deep radar layers in ice streams $(\mathrm{Ng}$ and Conway, 2004). Examples of the structures described here can be found in Figure 1a.

Given that we know the folds form from the cumulative effect of flow and submergence, what mechanisms govern their structure? And can we understand them without simulation? We advance a theory of folding that invokes the slope surface.

\subsubsection{Isochrone slopes on a radargram}

A systematic study of folding might begin by identifying the regions of positive and negative isochrone slopes on a radargram. In Figure 1a, for example, separate domains occur where the isochrones descend with distance $(s>0)$ and where they ascend with distance $(s<0)$ (remember the slope $s=\partial z / \partial x$ and $z$ points downward). On each isochrone, the crests and troughs occur where $s=0$; we call these points 'fold hinges' (Fig. 2), although geologists reserve the term for points on a layer where it attains maximum curvature. The isochrone curvature $(\partial s / \partial x)$ at a hinge is negative, positive or zero, respectively, if the hinge lies at the trough of a down-pointing fold (syncline), the crest of an uppointing fold (anticline) or the saddle of an inflexion fold. Fold limbs are portions of an isochrone that connect neighbouring hinges, and the locus of hinges across adjacent isochrones forms a hinge line (Fig. 2). Hinge lines mark the boundaries between the domains of opposite isochrone slopes. Although we have used the symbols $x, z$ and $s$ loosely in reference to Figure 1 a without first transforming its axes, the morphological features considered here can be defined in the same way for transformed radargrams.

Crucially, these features have equivalent representations on the slope surface $s(x, t)$. As Figure 4 shows, hinge lines occur where this surface intersects the horizontal plane $s=0$ (they are zero-slope contours), and the domains $s>0$ and $s<0$ are defined, respectively, by where the surface lies above and below the plane. Thus, like contours on a topographic map, hinge lines are expected to form closed loops unless they meet the edge of the radargram (notably $t=z=0$, where $s \equiv 0$ ). This is also true for loci that trace any constant value of slope.

The mechanisms determining the distribution of isochrone slopes on a radargram may now be explained. Given the accumulation rate $a(x)$ and flow velocity $u_{0}$, slope propagation along characteristics on the $x-t$ plane (Equation (14)) governs the shape of the slope surface $s(x, t)$, which in turn governs the distribution of slopes and position of hinge lines on that plane. If $a(x)$ fluctuates to make $b(x)$ $(=\mathrm{d} a / d x)$ flip between positive and negative, then Equation (15) predicts $s$ to rise and fall along characteristics, and the slope surface will be wavy, with zero-crossings that form a system of hinge loops (Fig. 4). The final step involves transforming $s(x, t)$ to the radargram domain (the $x-z$ plane), with $z(x, t)$ from Equation (13) telling us at what depth to place each slope value. Accordingly, hinge lines on the radargram are distorted from their counterparts on the $x-t$ plane, but retain the latter's topology.

An example of mapping between isochrones on the $x-Z$ plane and their slopes on the $x-t$ plane is given in Figure 5, where we show selected isochrones of a fold structure from 


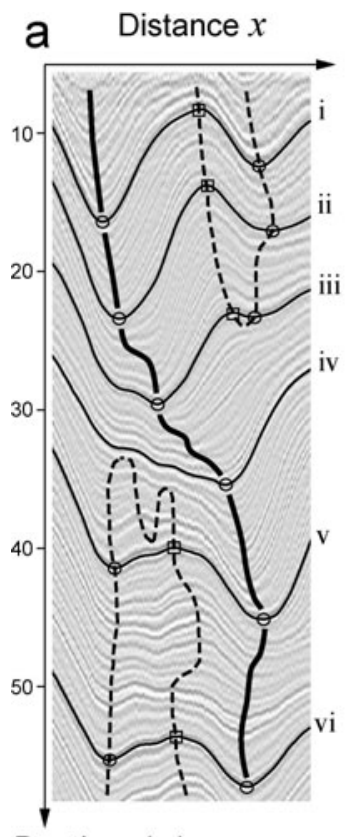

Depth $z(\mathrm{~m})$

\section{b Isochrone slope $s$}

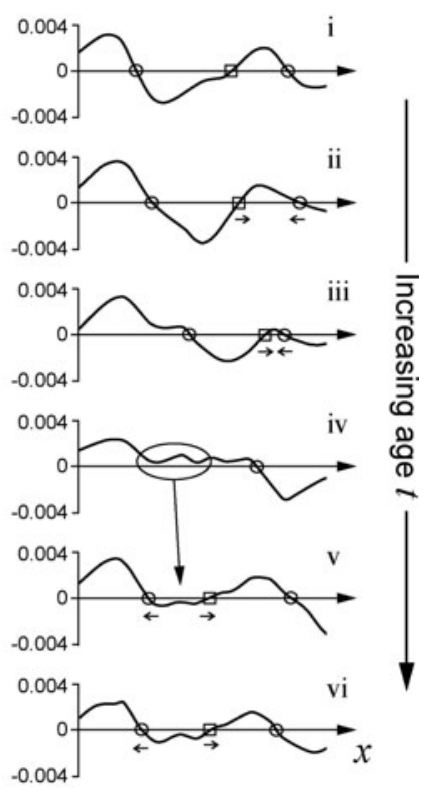

Fig. 5. (a) Isochrones of a fold structure from the white box in Figure $1 \mathrm{a}$ and (b) variation of their slope with distance $x$. The isochrones are numbered $\mathrm{i}$ to vi in order of increasing age $t$. In (a), the bold curve traces the hinge line of a trough that persists with depth, and dashed curves identify two hinge loops. Squares and circles locate the crests and troughs of isochrones, respectively. Arrows in (b) indicate the hinge transitions discussed in the text.

Figure 1a alongside plots of their slope against $x$. To see how $s$ varies with $t$, we order these plots to reflect the increasing age of the isochrones with depth (their absolute ages are unknown). The main structure is a fold trough that tilts down-glacier as it descends. Two other structures are visible. In the top $25 \mathrm{~m}$, right of the main fold, is a pair of anticline and syncline that gradually shallow and disappear as they descend. Figure 5b shows that their crest and trough points approach each other and cancel on contact and that this merging occurs via an inflexion point (where $s=\partial s / \partial x=0$ ). Left of the main fold, below $30 \mathrm{~m}$, we see the reverse behaviour, with a syncline-anticline pair and corresponding pairs of crest and trough points being created via an inflexion.

These phenomena, which we term 'hinge transitions', are a consequence of the fact that hinge lines manifest looped contours of a theoretical surface. As Figure 5 indicates, the first kind of transition occurs at the lower end of a loop, where two neighbouring hinge lines with opposite signs of $\partial s / \partial x(\mathrm{a}$ trough followed by a crest or vice versa) meet and terminate. The second kind of transition occurs at the top end of a new loop, where two hinge lines of opposite signs emerge. These insights stress the importance of tracing hinge loci along fold crests as well as along troughs. We examine the hinge-andslope structure of other radargrams in Section 3.

\subsubsection{Slope and hinge migration}

We proceed to derive mathematical results for the configuration of fold-hinge loci in this theory, considering what happens on both the $x-t$ and $x-z$ planes.

Earlier we solved Equation (14) to see how $s$ varies along the characteristics. But we can analyse slope propagation differently, by asking: along what trajectory does constant

slope propagate? Rearranging Equation (14) as

$$
\frac{\partial s}{\partial t}+\left[u_{0}-\frac{b(x)}{\partial s / \partial x}\right] \frac{\partial s}{\partial x}=0,
$$

we can write

$$
\frac{\mathrm{d} s}{\mathrm{~d} t}=\frac{\partial s}{\partial t}+\left.\frac{\mathrm{d} x}{\mathrm{~d} t}\right|_{s=\text { const }} \frac{\partial s}{\partial x}=\frac{\partial s}{\partial t}+v(x, t) \frac{\partial s}{\partial x}=0
$$

where

$$
\left.\frac{\mathrm{d} x}{\mathrm{~d} t}\right|_{s=\text { const }}=v(x, t)=u_{0}-\frac{b(x)}{\partial s / \partial x} .
$$

On the $x-t$ plane, curves of constant $s$ are therefore defined by $\mathrm{d} x / \mathrm{d} t=v(x, t)$, meaning that, as we cross isochrones of increasing age, each constant slope value propagates downflow at velocity $v$-we call this the slope migration velocity. Offset between $v$ and the material velocity $u_{0}$ is expected because we are dealing with kinematic waves: as each piece of an isochrone is advected by ice flow, its slope becomes modified by the local gradient in the surface accumulation rate. Equation (20) shows that the offset is proportional to this gradient, $b(x)$, and inversely proportional to the isochrone curvature, $\partial s / \partial x$.

Since Equation (20) holds for any constant value of $s$, we can use it to predict the hinge migration velocity - how fast hinge points $(s \equiv 0)$ move down-flow as they age. For trough points (where $s=0$ and $\partial s / \partial x<0$ ), this velocity exceeds $u_{0}$ if $b>0$ and is less than $u_{0}$ if $b<0$ (we call these 'supercritical' and 'subcritical' cases, respectively), whereas for crest points (where $s=0$ and $\partial s / \partial x>0$ ) the effect of $b$ is opposite. Equation (20) also predicts $v$ to be negative if $b /(\partial s / \partial x)>u_{0}$. These results explain why some hinge lines dip forward and others backward, why their dips do not indicate $u_{0}$ directly and why it may be crude to interpret the migration velocities indicated by them to be always less than $u_{0}$. In this regard, Equation (20) shows that $v$ can become large for small $\partial s / \partial x$ and, in fact, undefined at inflexion points (where $\partial s / \partial x=0$, i.e. at hinge transitions). Consequently, when tracing fold hinges on a radargram, one should not trace only the steeply dipping loci and neglect the shallow-dipping loci, which are typically less conspicuous.

Next we use the velocity $v$ to predict the shape of hinge lines on the radargram. As an isochrone evolves over an age increment $\delta t$, a point on it with constant slope migrates down-glacier by the distance $\mathrm{d} x /\left.\mathrm{d} t\right|_{s=\text { const }} \delta t=v(x) \delta t$, but lowers by the height $\mathrm{d} z /\left.\mathrm{d} t\right|_{s=\text { const }} \delta t$, where

$$
\begin{gathered}
\left.\frac{\mathrm{d} z}{\mathrm{~d} t}\right|_{s=\text { const }}=\frac{\partial z}{\partial t} \\
+v \frac{\partial z}{\partial x}=\frac{\partial z}{\partial t}+\left[u_{0}-\frac{b(x)}{\partial s / \partial x}\right] \frac{\partial z}{\partial x} \\
=a(x)-s \frac{b(x)}{\partial s / \partial x} .
\end{gathered}
$$

(We have used Equation (10) and the definition $s=\partial z / \partial x$.) On the $x-z$ plane, the point thus descends in a direction determined by the ratio $\mathrm{d} z /\left.\mathrm{d} t\right|_{s=\text { const }}: \mathrm{d} x /\left.\mathrm{d} t\right|_{s=\text { const }}$. At hinge points $s \equiv 0$, so

$$
\left.\frac{\mathrm{d} z}{\mathrm{~d} t}\right|_{s=0}=\mathrm{a}(x) .
$$

Combining this with Equation (20) yields

$$
\left.\frac{\mathrm{d} x}{\mathrm{~d} z}\right|_{s=0}=\frac{\mathrm{d} x /\left.\mathrm{d} t\right|_{s=0}}{\mathrm{~d} z /\left.\mathrm{d} t\right|_{s=0}}=\frac{v(x, t)}{\mathrm{a}(x)}=\frac{u_{0}-\frac{b(x)}{\partial s / \partial x}}{a(x)} .
$$

This result shows that the local dip of a hinge line depends 

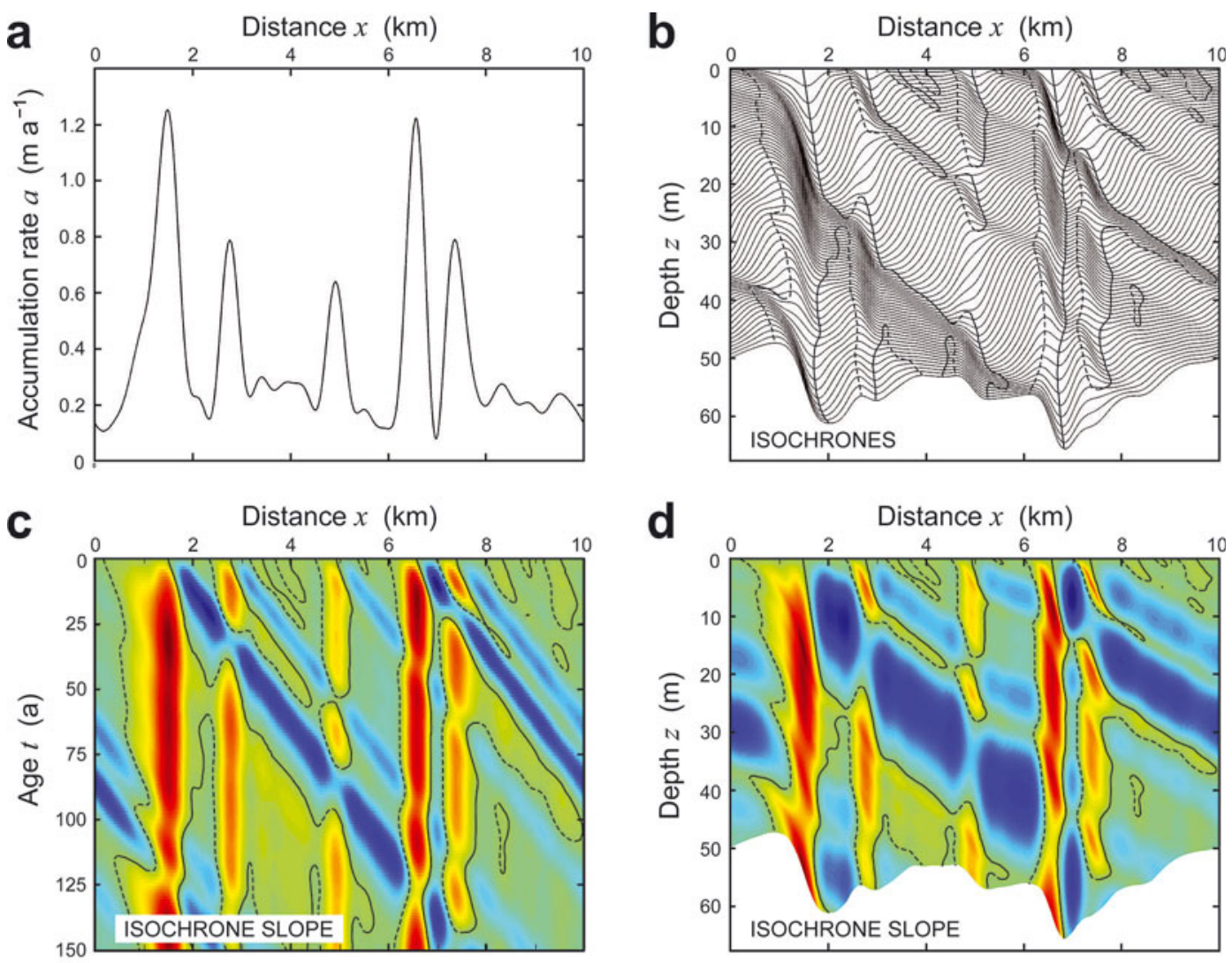

Slope

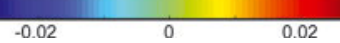

Fig. 6. Analysis of a synthetic radargram. (a) Prescribed accumulation rate pattern. (b) Synthetic radargram made from model-simulated isochrones with a constant age increment of 2.5 years. (c) Isochrone slope map on the $x-t$ plane. (d) Isochrone slope map on the $x-z$ plane. In (b-d), solid and dashed curves trace the hinges of fold troughs and fold crests, respectively.

on the flow velocity $u_{0}$, the local accumulation rate and its spatial gradient, and the curvature of the isochrone at the hinge.

Equations (20) and (23) are differential equations for the hinge-line trajectories $(x(t)$ and $x(z))$ on the $x-t$ and $x-z$ planes. Their similar form is not surprising given the link between the isochrone slope distributions on these planes; but since $\partial s / \partial x$ enters both equations, we cannot solve for the trajectories without knowing these distributions. In both equations, the term representing the velocity offset is negligible if $|b| \ll|\partial s / \partial x|$, meaning that slope and hinge migration should occur at close to the 'critical' (material) velocity $u_{0}$ where the accumulation rate varies slowly with distance. We will see examples of this behaviour in the case studies in Section 3.

\subsubsection{Hinge-line systems}

We can now anticipate the general architecture of hinge lines on a steady-flow radargram. If the accumulation rate pattern undulates, then hinge lines extend into the firn from the surface at stationary points of $a(x)$ (where $b=0$ ). Alternation of these points between minimum and maximum causes successive hinges to switch between crests and troughs. As hinge lines descend, their trajectories depend predictably on ice-flow and accumulation forcings (Equation (23)) and may locally express supercritical or subcritical (even negative) migration velocities.

Given their inception as contours of the slope surface, hinge lines are also linked globally across the radargram.
They do not start or end on their own, but emerge in (cresttrough) pairs at the top of hinge loops or vanish in pairs by merging at the bottom of hinge loops. Equation (16b) yields further insights into their geometry on the $x-t$ plane. It predicts $s=0$ when $a(x)=a\left(x-u_{0} t\right)$ so that, on a given isochrone aged $t$, hinges occur wherever the local accumulation rate equals the accumulation rate at a distance $u_{0} t$ upflow. This makes sense because each piece of isochrone has a slope that represents the integrated history of slope alteration by $b(x)$ over its journey to the present position.

\section{CASE STUDIES}

In this section we analyse two radargrams - one synthetic and one real, both aligned with the ice flow - to test and further explore the theory. Concepts from Section 2 are used to identify structure behind their stratigraphic undulations. We also develop a way of extracting accumulation rates from stationary isochrone patterns that lack age information.

\subsection{Synthetic radargram}

We first use the forward model to create a radargram for which everything is known. In this example, we assume the accumulation rate pattern in Figure $6 a$, and we ignore firn densification and assume a constant ice-flow velocity $u=u_{0}=40 \mathrm{~m} \mathrm{a}^{-1}$, which allows us to work directly in the transformed distance and depth coordinates. With these steady forcings, Equation (10) is solved numerically by finite difference (with upwinding and explicit time-stepping) to 


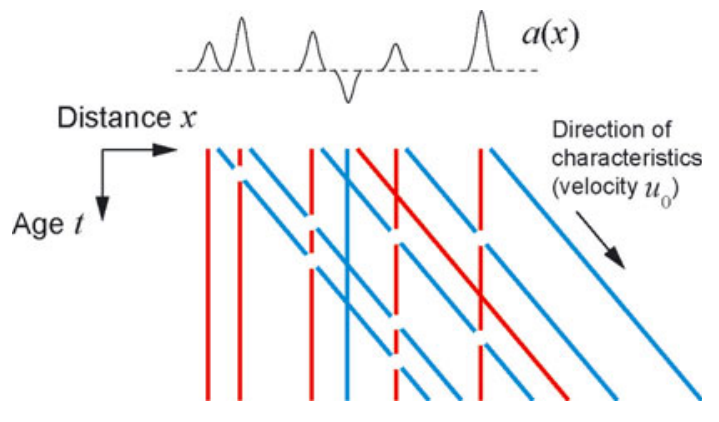

Fig. 7. Schematic of the criss-cross arrangement of distinct vertical and diagonal zones on the $x-t$ plane of a steady-flow radargram, as caused by anomalies in accumulation rate $a(x)$ (cf. Figs $6 \mathrm{c}$ and $10 \mathrm{~g}$ ). Red and blue zones signify where isochrones plunge and rise, respectively. Constructive superposition of layer slopes occurs where same-coloured zones intersect, reinforcing each other's slope contributions. Destructive superposition of layer slopes occurs where different-coloured zones intersect and offset each other's contributions.

evolve an isochrone from the surface, thus generating a sequence of isochrones with an age increment of 0.025 years. Edge effects were avoided by assuming the $10 \mathrm{~km}$ horizontal domain to be periodic; we halted the simulation at $t=150$ years before this caused the isochrone pattern to repeat at depth. The isochrones were subsampled at 2.5 year intervals to form the radargram, which is shown in Figure 6b.

As expected, down-pointing folds develop in the shallow subsurface under major peaks in the surface accumulation (see Fig. 6a and b). Deeper down, however, the undulating pattern becomes less predictable and shows a level of complexity like that shown in Figure 5.

To study the fold pattern, we traced hinge lines across the radargram by linking the stationary points of isochrones found by differentiating their depth (which also yields their slope). Trough and crest lines, distinguished in Figure $6 \mathrm{~b}$ by different line types, display a topology confirming our predictions. Despite their irregular shape they form loops, dividing the radargram into jigsaw areas of opposite isochrone slopes; the loops are closed unless they meet the surface. Hinge transitions of the kinds described in Section 2.4.1 are abundant. The upper/lower end of each loop locates where a pair of crest and trough lines emerges/ vanishes; such pairing causes hinge lines to switch between crests and troughs in the $x$-direction. At hinge transitions, the trough and crest lines join smoothly with zero gradient and do not form cusps. We know from our theory that this is because the underlying slope surface is smooth.

Different portions of the hinge lines in Figure $6 \mathrm{~b}$ show a variety of dips, some pointing up-flow but more often downflow (cf. Arcone and others, 2005). Two dips are common. One of them is nearly vertical. The other dip has a downglacier slope of $\sim 0.01$, as shown by several loops that swing towards the lower right. This dip seems to track the mean direction of advection coupled with burial, since material moves down-glacier by $6 \mathrm{~km}$ in the time it takes to reach the depth $(\sim 60 \mathrm{~m})$ of the oldest isochrone ( $t=150$ years). Both dips here are associated with the zones of steep isochrone slope discussed below.

We examine the folds more generally by considering isochrone slope $s$, which varies with $x$ and $z$ on the radargram, but, as explained in Section 2, may be conceived to vary with $x$ and $t$. Figure $6 \mathrm{c}$ and d plot the functions $s(x, t)$ and $s(x, z)$ in colour. These 'slope maps' are easy to compile for this radargram because we know the age and depth of its isochrones. Features on $s(x, z)$ are distorted versions of those on $s(x, t)$, but can be superposed directly on the stratigraphy in Figure 6b.

We focus on $s(x, t)$ (Fig. 6c) because it shows the essence of slope information travel. Intriguingly, we see two distinct directions of constant-slope migration, shown by the vertical zones of red and diagonal zones of blue, both descending from major accumulation anomalies (cf. Fig. 6a). The red is aligned with peaks in $a(x)$; the blue emanates from the lee of these peaks and inclines at $\sim 40 \mathrm{~m} \mathrm{a}^{-1}\left(u_{0}\right)$. As these colours respectively signify strongly positive and strongly negative slopes, their pattern means that steep slopes on the descending flanks of layer folds migrate at velocity $v \approx 0$, whereas steep slopes on the ascending flanks of layer folds migrate at $v \approx u_{0}$.

This pattern is explained by slope evolution along the characteristics (defined in Section 2.2), which descend across the $x-t$ plane at velocity $u_{0}$. Equation (15) shows that on this plane, $s$ depends on its initial value on its characteristic (at $t=0$ ) and on its alteration by $b(x)$ along the characteristic. The observed pattern stems from the contest between these inheritance and modification factors, as may be understood by considering two end-member scenarios. First, slopes that are shallow initially (of either sign) can be changed easily by a strong accumulation anomaly on their course, to adopt its sign at its $x$-position. Thus a major peak or valley in $a(x)$ causes isochrones beneath it to plunge or rise steeply, respectively, forming a vertical zone of high slopes (e.g. red in Fig. 6b). Second, an initially steep slope (of either sign) inherited from a strong accumulation anomaly will stay steep unless strong opposite anomalies lie on its characteristic to negate it. In this case, a set of steep fold limbs drifts down-flow with little slope change to form a diagonal zone; an example in Figure $6 \mathrm{~b}$ is the rising limbs between $x=2 \mathrm{~km}, z=10 \mathrm{~m}$ and $x=6 \mathrm{~km}$, $z=45 \mathrm{~m}$.

These scenarios together explain why each accumulation anomaly creates a pair of vertical and diagonal zones. Moreover, since our wave equation is linear, where these zones intersect they disrupt each other by partial cancellation of layer slopes (Fig. 6c). The current analysis identifies such spatial arrangement, which we summarize in Figure 7 , as the hallmark of layer patterns formed under steady forcings. We learn that although the layer folds in different steady-flow radargrams may look distinct, they obey the same general rules of patterning, with the main difference being the location and size of accumulation anomalies. We also learn the usefulness of the slope map $s(x, t)$ as a tool for elucidating the structure of these folds.

\subsection{Extracting $a(x)$ and layer ages through optimization}

Compiling the map $s(x, t)$ for any radargram requires the age of its layers to be known. Here, making further use of the synthetic radargram, we devise a method (based on method I in Section 2.3) capable of retrieving the accumulation rate $a(x)$ and layer ages simultaneously from steady-flow radargrams.

Given the depths of two adjacent isochrones, $z_{1}(x)$ (shallower) and $z_{2}(x)$ (deeper), we can use Equation (17) to estimate $a(x)$ by means of three steps: 

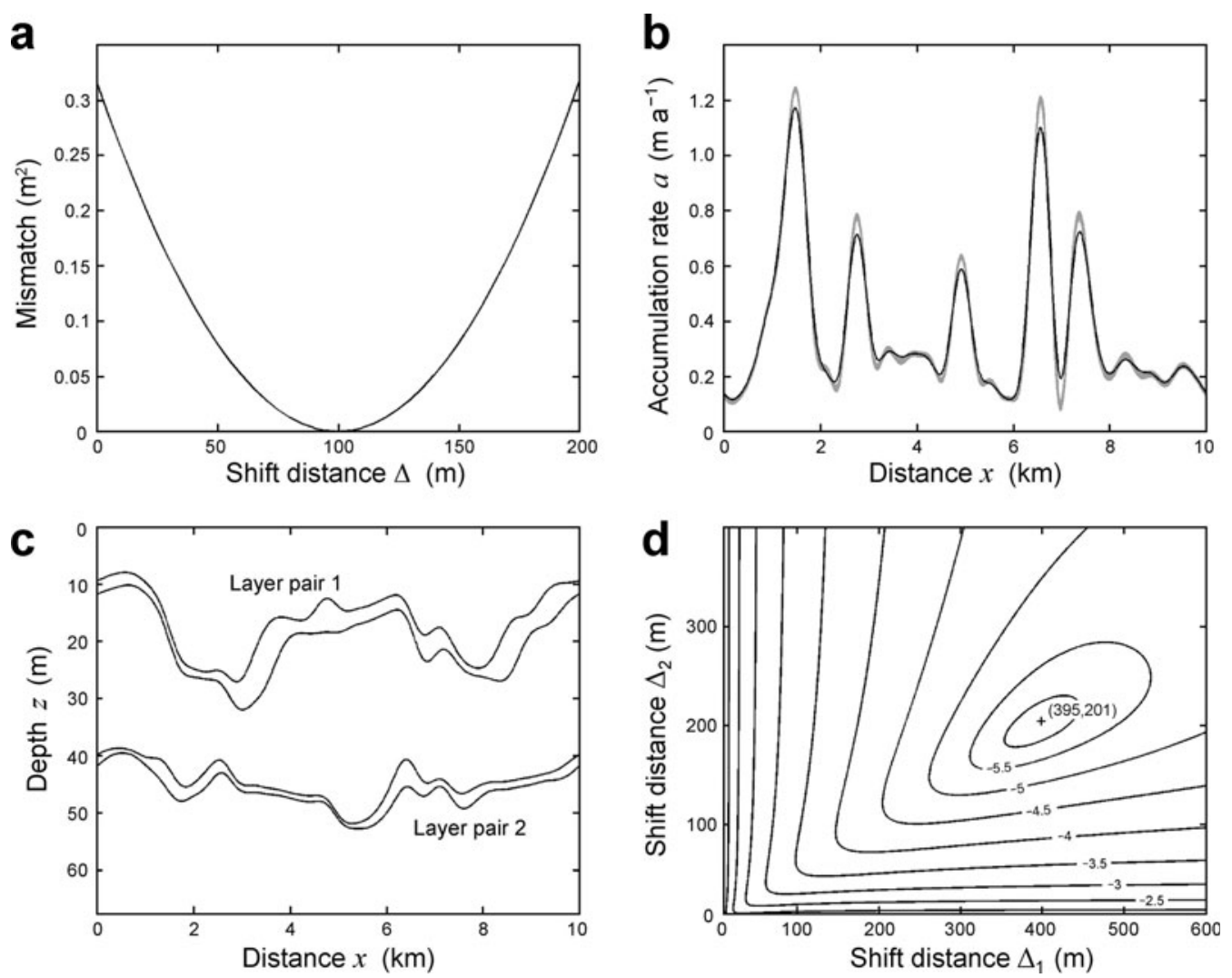

Fig. 8. Retrieval of accumulation rate from the synthetic radargram layers in Figure 6b. Results of two methods are presented. (a) Mismatch between $\delta z$ profiles derived from shift-differencing all consecutive layers by the same shift distance $\Delta$, plotted against the value of $\Delta$. The point of minimum mismatch identifies the optimal shift. (b) Retrieved accumulation rate patterns: grey curves show the results of the method in (a), the black curve the result of the method in (c) and (d). (c) Layer pairs chosen to illustrate the method in Figure 9. (d) Mismatch (log-10 scale) across the parameter space of shift distances $\Delta_{1}$ and $\Delta_{2}$. The combination of $\Delta_{1}$ and $\Delta_{2}$ with minimum mismatch yields the black curve in (b).

1.

2.

$$
\delta z=z_{2}(x+0.5 \Delta)-z_{1}(x-0.5 \Delta)
$$

3.

$$
\begin{gathered}
\delta t=\Delta / u_{0} \\
a(x) \approx \delta z / \delta t .
\end{gathered}
$$

These steps involve 'shift-differencing' the layers (by the shift distance $\Delta$ ), inferring their age difference $\delta t$ from $\Delta$, and finding the accumulation rate by dividing the depth difference $\delta z$ by $\delta t$. As before, $x$ and $z$ here are transformed coordinates.

In scenarios where either $\delta t$ or $u_{0}$ is unknown, the correct shift distance $\Delta$ to use in step (1) is unknown. However, given multiple pairs of isochrones, we can choose a combination of $\Delta^{\prime}$ 's for these pairs to optimize the agreement between the $a(x)^{\prime}$ 's reconstructed from them, thus also determining the $\Delta^{\prime}$ 's. In this method, a key assumption is that the isochrone pattern is stationary, generated by steady forcings.

In an experiment, we tested this method by using all 61 layers of the synthetic radargram (Fig. 6b) and using the knowledge that their age difference is uniform so that the same $\Delta$ can be used in step (1) to shift-difference all consecutive layer pairs. For each choice of $\Delta$, this step yielded 60 difference profiles (the $\delta z^{\prime} \mathrm{s}$ ). We then calculated the variance of these $\delta z^{\prime}$ s at each $x$-position and used the spatial mean of this variance as a single measure of mismatch between the difference profiles. Figure 8a shows that the mismatch is minimized (agreement between the difference profiles maximized) when $\Delta=100 \mathrm{~m}$. With our additional knowledge of $u_{0}=40 \mathrm{ma}^{-1}$ in the synthetic problem, this optimal shift distance correctly recovers $\delta t=2.5$ years in step (2). The corresponding estimates of $a(x)$, found from step (3) and plotted in grey in Figure $8 b$, match the actual accumulation pattern in Figure 6a closely, despite a small scatter due to truncation error.

In practice, many radargrams are not furnished with local firn cores to constrain the age of their stratigraphy (e.g. via annual-layer counting); when 'picking' layers from them there is no way to ensure uniform age difference between the picks. But we can circumvent this requirement because steps (2) and (3) in Equation (24) can be combined to give

$$
a(x) \approx u_{0}\left(\frac{\delta z}{\Delta}\right) .
$$

Consequently, when shift-differencing multiple layer pairs to find $a(x)$, we can minimize the mismatch between profiles of $\delta z / \Delta$ rather than of $\delta z$. The optimization now involves choosing as many $\Delta^{\prime}$ s as there are layer pairs, not just one $\Delta$.

We tested this method on the synthetic radargram taking only the two pairs of isochrones shown in Figure 8c. Figure 9 illustrates the shift-differencing procedure on the $x-t$ plane. Two shift distances $\Delta_{1}$ and $\Delta_{2}$ were chosen to difference the pairs to yield two estimated profiles of $\delta z / \Delta$, whose variance was summarized as a mismatch in the same way as before. The best combination, $\Delta_{1}=395 \mathrm{~m}$ and $\Delta_{2}=201 \mathrm{~m}$, was found by searching for minimum mismatch over the parameter space (Fig. 8d). The corresponding reconstructed $a(x)$ is the black curve in Figure $8 \mathrm{~b}$. It is 


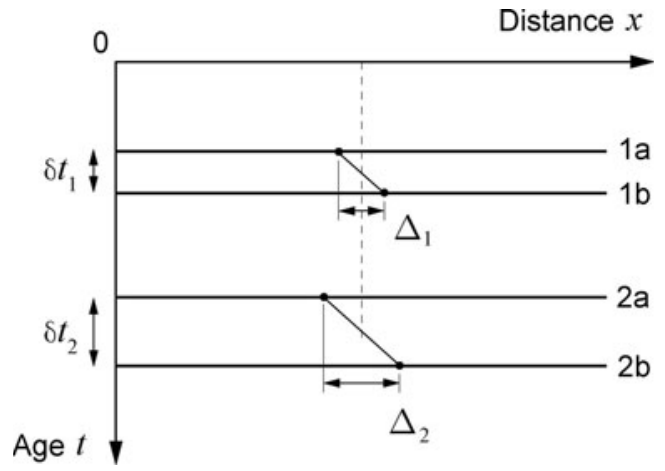

Fig. 9. The procedure (on the $x-t$ plane) of shift-differencing two pairs of isochrones whose age differences are unknown and unequal.

smoother than the original accumulation forcing, indicating that truncation error associated with the separation between the input layers causes low-pass filtering. Nevertheless, with only four isochrones as input, this reconstruction recovers the peaks and troughs of the forcing remarkably well.

Besides its ability to retrieve accumulation rates, this method has three useful properties that are worth noting:

1. The optimal shift distance $\Delta$ for each pair of layers divided by the flow velocity $u_{0}$ gives their age difference (step (2) in Equation (24)).

2. Even if $u_{0}$ is unknown, the method can reconstruct the normalized accumulation rate pattern, $a(x) / u_{0}$ (see Equation (25)).

3. Since the method assumes steady forcings, layer patterns formed by $a$ and $u$ that varied temporally as well as spatially (so that $a / u_{0}$ is not invariant) will cause fundamental mismatch between the $\delta z / \Delta$ profiles from different layer pairs that cannot be reconciled by any shift combination. In this sense, the method is able to discern the existence of unsteadiness, although not quantify its history.

\subsection{Radargram from Bindschadler Ice Stream}

Our real case study uses the radargram in Figure 1, obtained by ground-based radar on a traverse at the onset zone of Bindschadler Ice Stream in the 2001/02 austral summer. Along the $52.7 \mathrm{~km}$ radar line, surface velocity increases from 59 to $111 \mathrm{~m} \mathrm{a}^{-1}$. Radar traces were recorded by a $100 \mathrm{MHz}$ pulseEKKO radar at $0.8 \mathrm{~ns}$ time interval and every $5 \mathrm{~m}$ along flow. Conversion of two-way travel time to depth used the wave speed in firn measured by a common-midpoint survey at $\mathrm{km} 33$, which shows that this speed decays smoothly from $\approx 0.23 \mathrm{~m} \mathrm{~ns}^{-1}$ at the surface to a constant value of $\approx 0.168$ $\mathrm{m} \mathrm{ns}^{-1}$ below a depth of $50 \mathrm{~m}$, consistent with an expected increase of firn density with depth. Migration was found to cause negligible change to the depth and slope of isochrones and so was ignored. For details of radar processing, see Woodward and King (2009).

The modern ice-flow dynamics of this region have been characterized by field and remote-sensing observations and numerical modelling (e.g. Joughin and Tulaczyk, 2002; Price and others, 2002), but little is known about their history over centuries or longer. For Bindschadler Ice Stream, interpretation of its deep radar layers (Siegert and others, 2003) and of features downstream of it on the Ross Ice Shelf (Hulbe and
Fahnestock, 2007) does not suggest that its flow has undergone major fluctuations. However, deep radar-layer folds upstream of the onset zone have been interpreted for changes in flow direction there $\sim 1.5 \mathrm{ka}$ ago (Siegert and others, 2004).

We picked the depth profiles of 17 clearly visible layers in Figure $1 \mathrm{a}$ and smoothed them by low-pass Fourier filtering to suppress noise for the later slope calculations. In view of the flow acceleration along the radar line, we first rendered the profiles in the transformed coordinates $x$ and $z$ (Section 2.1). We used the linear velocity model in Equation (8) with $u_{0}=59 \mathrm{~m} \mathrm{a}^{-1}$ and $k=0.0167 \mathrm{~km}^{-1} ; x$ is then related to true distance via Equation (9a). For the firn-densification correction in Equation (3), we assumed the density profile $\rho(z)=\rho_{\mathrm{i}}-\left(\rho_{\mathrm{i}}-\rho_{0}\right) \mathrm{e}^{-c z}$ with $\rho_{\mathrm{i}}=917 \mathrm{~kg} \mathrm{~m}^{-3}, \rho_{0}=400 \mathrm{~kg}$ $\mathrm{m}^{-3}$ and $c=1 / 35 \mathrm{~m}^{-1}$ (Arcone and others, 2005) derived from International Trans-Antarctic Scientific Expedition (ITASE) Core 99-1, the nearest firn core $(\approx 25 \mathrm{~km}$ upstream) from our line (Fig. 1b). Figure 10b shows the distance conversion and Figure 10a plots the layers after transformation, which squashes them laterally and depresses them towards the right-hand side of the domain.

Of interest are the hinge-slope structures of the layer pattern and their links with the accumulation rate pattern. Unlike in the synthetic study, however, here $a(x)$ is not known, nor are layer ages needed for compiling the slope map $s(x, t)$. We estimated these by the inversion method developed in Section 3.2, assuming the picked layers to be isochronal and their pattern stationary.

This problem is more complicated than the example in Figure 8c because we need to choose 16 shift distances (one $\Delta$ for each pair of consecutive layers) to maximize the agreement between the $\delta z / \Delta^{\prime}$ 's found from shift-differencing the respective layer pairs. We determined the $\Delta^{\prime}$ s in two steps: by finding rough guesses and refining them. In the first step, the method in Figure 9 was applied to two pairs of consecutive layers at a time, but to all 15 consecutive pairs of layer pairs. For the top- and bottommost layer pairs this yielded the $\Delta$ guess directly; for the other layer pairs this yielded two estimates of $\Delta$, which we averaged to form the guess. In Figure 10c, the grey curves plot the normalized accumulation profiles $a(x) / u_{0}$ evaluated by Equation (25) with these $\Delta$ guesses. Although they look shifted vertically from each other, their peaks and valleys show correlation.

In the second step, we adjusted the $\Delta$ guesses to optimize the agreement between the $\delta z / \Delta$ profiles found from shiftdifferencing all 16 layer pairs together; accordingly we redefined the measure of mismatch based on all these profiles. The optimization used a Monte Carlo scheme with many trials (Press and others, 1992) and the $\Delta$ guesses as starting point. In each trial, we chose a pair of consecutive layers at random and improved its $\Delta$ by minimizing the mismatch, keeping the other $\Delta^{\prime}$ s fixed. This procedure brought different estimates of $a(x) / u_{0}$ into focus without the need for exhaustive search on the 16-dimensional space of the $\Delta^{\prime}$ s. The mismatch decayed rapidly at first and stabilized at $\approx 7 \times 10^{-4} \mathrm{~m}^{2}$ after about 100 trials. Figure $10 \mathrm{~d}$ shows the optimal shift distances. As expected, these distances are larger for layer pairs that lie further apart. Since the edge of this radargram caused the $\delta z / \Delta$ profiles to be shorter than the domain, in both steps above we evaluated their variance (and $a(x) / u_{0}$ ) only where they exist and overlap.

In Figure 10e, the grey curves show the normalized accumulation rate profiles $a(x) / u_{0}$ calculated with the optimal shifts. Their broad agreement means that different 
a

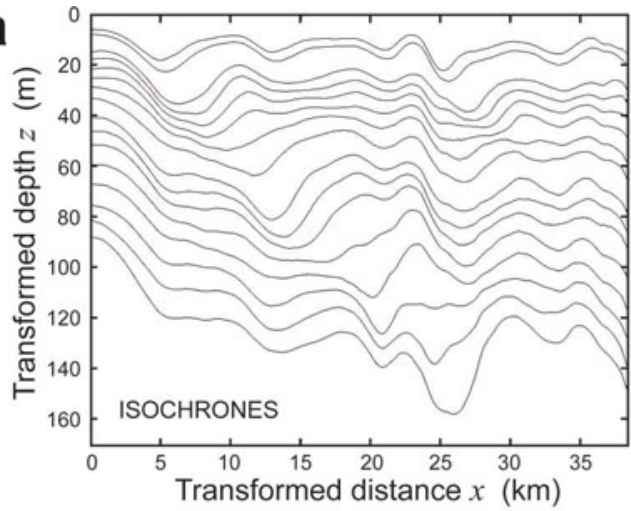

C

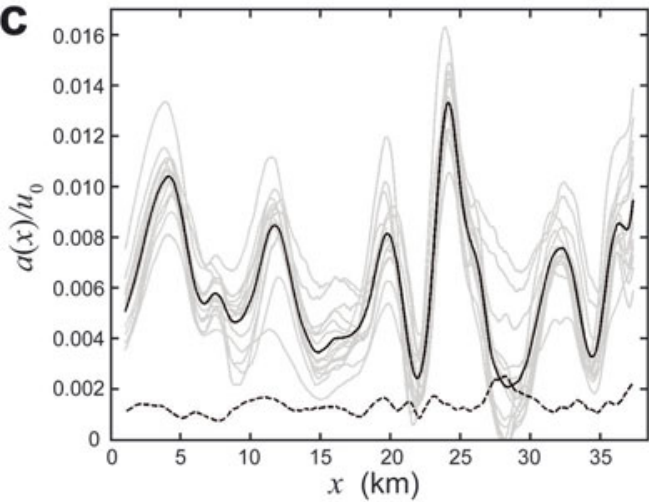

e

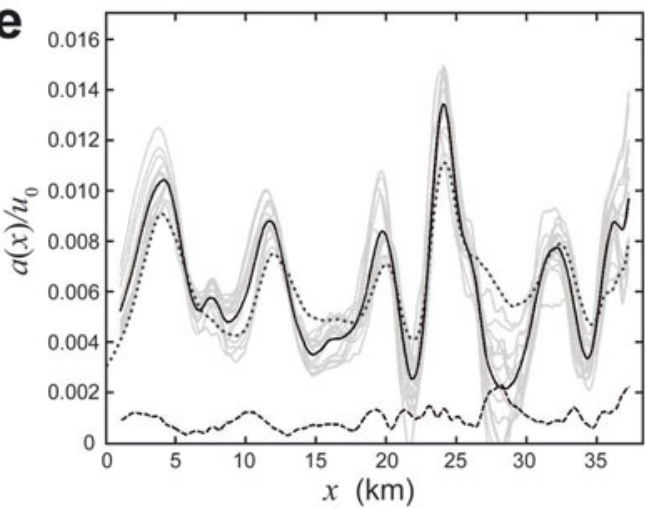

g

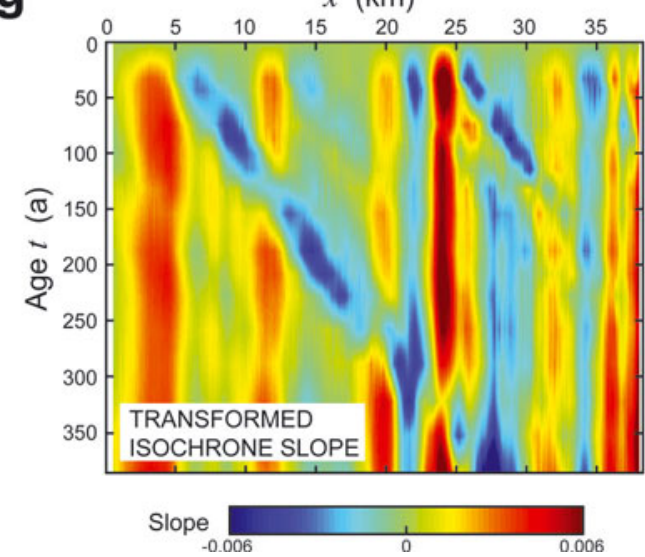

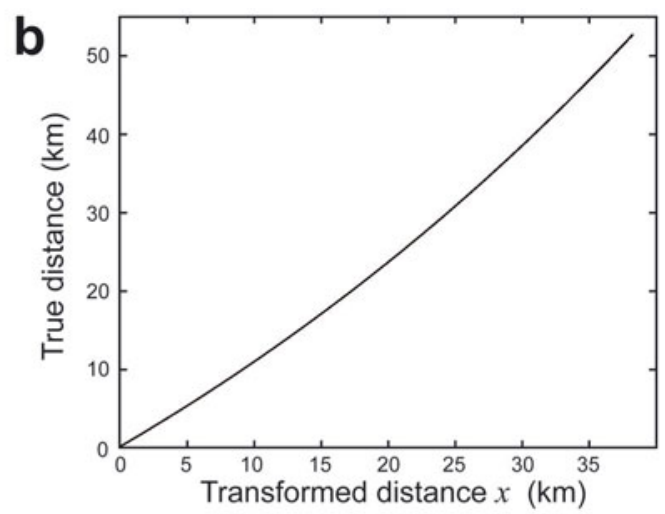

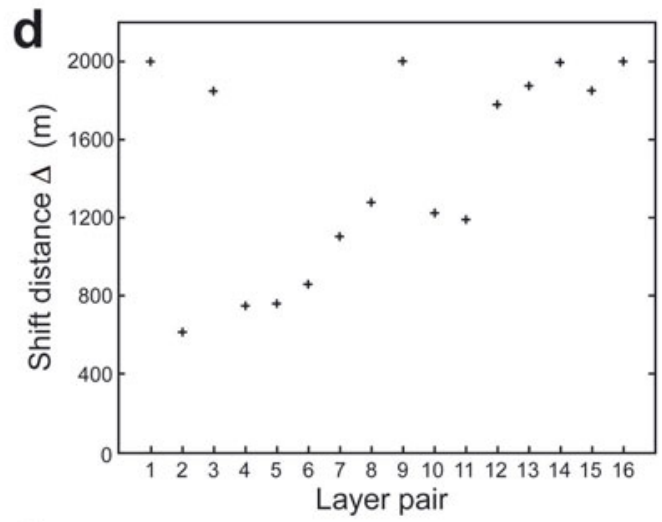

f

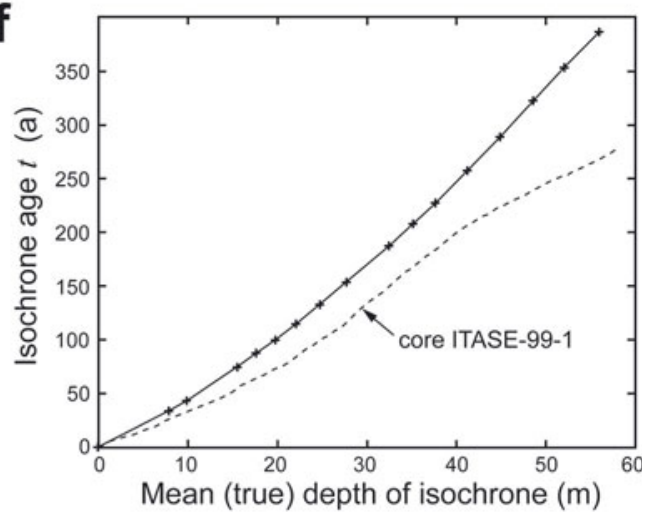

h

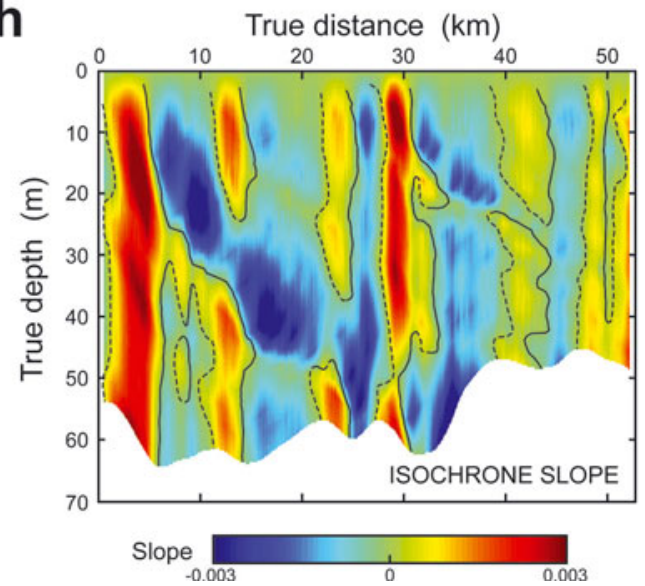

Fig. 10. Model analysis of the BAS Line 11 radargram in Figure 1a. (a) 17 picked isochrones (including the surface) in the transformed coordinates $x$ and $z$. (b) Conversion between transformed distance $x$ and true horizontal distance based on Equation (9a). (c) Initial estimates of the normalized accumulation rate from each of the 16 layer pairs (see step 1 of the method in Section 3.3). (e) Final estimates of the normalized accumulation rate (step 2 of the method) and (d) the corresponding optimized shift distances for the layer pairs. In (c) and (e), grey curves show estimates found from individual layer pairs, black curve shows the mean of these estimates, and dashed curve shows their standard deviation. Dotted curve in (e) shows $a(x) / u_{0}$ found by shift-differencing the shallowest subsurface layer against the surface layer according to the method in Equation (17); it overlaps the grey curve corresponding to this pair of layers. (f) Inferred age of each layer vs the mean of its true depth. The age-depth profile from ITASE Core 99-1 is included for comparison. (g) Map of isochrone slopes (in transformed coordinates) on the $x$-t plane compiled using the layer ages in (f). (h) Map of isochrone slopes on the untransformed radargram domain. Also shown are hinge loci of fold troughs (solid curves) and fold crests (dashed curves) traced from Figure 1a. 

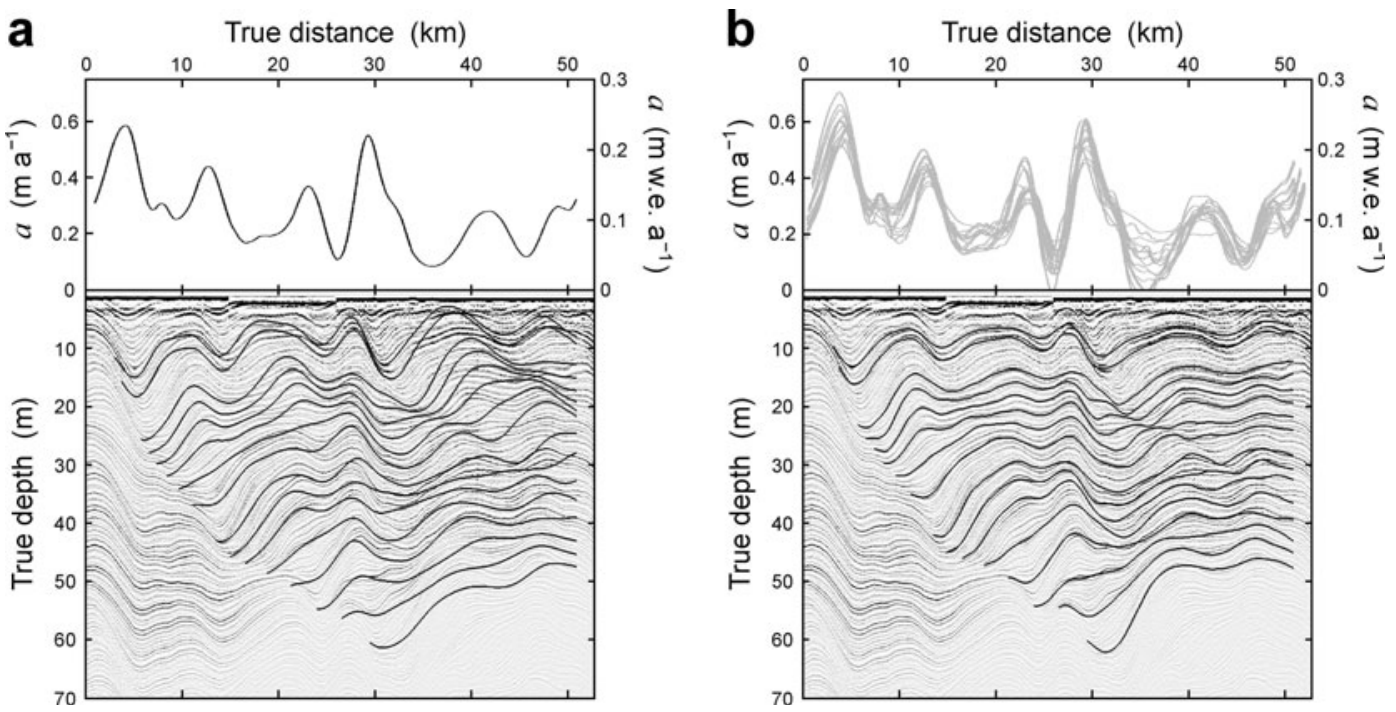

Fig. 11. Comparison of radar layers simulated by the forward model (curves on the lower plots) with those in the recorded radargram in Figure 1a (grey background in the lower plots). Upper plots show the accumulation rate forcings used in two different simulations: (a) the mean reconstructed $a(x)$ from Figure 10e; (b) the $a(x)$ 's reconstructed from individual layer pairs from Figure 10e. The accumulation rate scales are shown in both the units of velocity and water-equivalent units.

layer pairs tell similar stories about past accumulation; their scatter is worse than in the synthetic study but remains small compared with their mean. The scatter could arise from many factors. One factor is numerical errors from the layer picking, conversion of radar time to depth, and the truncation approximation in Equation (17). A second factor is processes unaccounted by our model, such as temporal changes in $u$ and $a$, lateral flow convergence or divergence, and spatial variability in densification. A third factor is geometries that render the radargram imperfect, including out-of-plane reflections and offset of the radar line from the ice-flow direction.

In some studies, the accumulation rate distribution was estimated from the depth and age of a shallow layer (e.g. Gray and others, 2005; Woodward and King, 2009). To see how well this method performs, in Figure 10e we plot the normalized accumulation rate found by shift-differencing our uppermost subsurface layer and the surface layer (see the dotted curve). The method in Equation (17) was used, with $u_{0} \delta t$ equated to the optimal shift distance of the layer pair (this effectively constrains the age of the lower layer). As our two-step optimization is also based on Equation (17) and the same shift, the two methods yield identical results of $a(x) / u_{0}$ (the dotted curve overlaps the grey curve for the layer pair). The shallow-layer result here resembles our mean optimized estimate for $a(x) / u_{0}$ (black curve in Fig. 10e) due to the limited scatter discussed above. However, when used on radargrams whose forcings have varied substantially, the shallow-layer method will not give valid accumulation estimates for times preceding the age of the lower layer.

We next converted all the optimal shift distances $\Delta_{\mathrm{i}}$ $(i=1-16)$ into age differences and summed these to estimate the age of the picked layers. We used the equation

$$
t_{m}=\sum_{i=1}^{m} \delta t_{i}=\sum_{i=1}^{m} \frac{\Delta_{i}}{u_{0}}
$$

where $m$ is the number of the layer and $t_{m}$ its age. Figure $10 \mathrm{f}$ plots the estimated age of each layer against its original (untransformed) mean depth on the radargram. This age-depth relationship gives older ages than found by layer counting in ITASE Core 99-1 (Steig and others, 2005), implying lower accumulation rates in our study area than at the core site tens of kilometres away. Indeed, our mean untransformed value of $a(x)$ is $0.273 \mathrm{~m} \mathrm{a}^{-1}$ or $0.109 \mathrm{~m}$ w.e. $\mathrm{a}^{-1}$ (Fig. 11a, top plot), which is $20 \%$ less than the mean accumulation rate $0.136 \mathrm{~m}$ w.e. $\mathrm{a}^{-1}$ derived for Core 99-1 over the period 1713-2000 (Dixon and others, 2004). Our age-depth relationship also shows an upward bend caused by firn densification, but we emphasize that it represents a spatial mean only. The local age-depth relationship must vary across the radargram because the radar layers undulate.

The slope maps $s(x, t)$ and $s(x, z)$ of this radargram (Fig. $10 \mathrm{~g}$ and $\mathrm{h}$ ) share strong similarities with those of the synthetic case study. The former map (Fig. 10g) was compiled using the layer ages estimated above and uses transformed distance as its horizontal axis. The latter map (Fig. 10h) uses true depth and true distance as axes. Both maps were made by interpolating the slopes of the 17 layers and without picking additional layers. As in the synthetic study, $s(x, t)$ here shows the criss-cross arrangement of vertical red and diagonal blue zones, descending respectively from the peaks and lee sides of accumulation anomalies. The blue zones in Figure $10 \mathrm{~g}$ are straightened from those in Figure $10 \mathrm{~h}$ and incline at a velocity near $u_{0}$. Compared with Figure $6 \mathrm{c}$ and d, both maps show more finescale features, some of which presumably stem from the variability that causes the minor differences between the retrieved profiles of $a(x) / u_{0}$ in Figure 10e.

These results imply that the layer pattern on this radargram can be explained by time-invariant forcings through our proposed folding mechanism. Agreement between $a(x) / u_{0}$ found from the different layer pairs reflects limited historical variations in this ratio and is consistent with the steady-flow assumption behind our analysis. If we use Equation (10) to forward-model the pattern with $u_{0}$ and the mean reconstructed $a(x)$ as inputs, and show the results in true coordinates, then isochrones simulated at the age of the picked layers are found to mimic the latter well in terms 
of the general location and structure of key folds (Fig. 11a). Improvement in matching the depth of isochrones is found if we use the $a(x)^{\prime}$ s reconstructed for the times spanned by successive layer pairs (Fig. 11b).

Inferences from these findings about the ice stream's history are non-unique because our model precludes temporal variations in $a$ and $u$ that might have occurred. Specifically, three different interpretations are possible:

1. Ice flow and accumulation conditions in the onset zone have been steady over a time going back to the age of the deepest picked isochrone (386 years in Fig. 10f). This interpretation implies centurial-scale ice-stream stability.

2. Both $a$ and $u$ changed over time, but co-varied in a way to maintain approximately the same $a / u_{0}$ ratio, producing an apparently stationary isochrone pattern.

3. The accumulation rate pattern has been stable but has been migrating up- or down-glacier, producing an apparently stationary isochrone pattern. This is possible if we suppose an accumulation forcing of the form $a(x-w t)$, where $w$ is the migration speed. Putting this forcing in our model of isochrone evolution in Equation (10), we see that the latter can be rewritten as

$$
\frac{\partial z}{\partial t}+\left(u_{0}-w\right) \frac{\partial z}{\partial x^{*}}=a\left(x^{*}\right),
$$

with $x^{*}=w t$ being a moving coordinate. As this equation has the same form as the original (albeit with advection velocity $u_{0}-w$ ), layer patterns generated by it will appear to have been caused by steady forcings when observed at a given time, but are actually translating at speed $w$ with respect to the stationary frame of reference. Migrating surface accumulation patterns not only could, but do, exist, as have been inferred for various dune-like features in East Antarctica (see review by Eisen and others, 2008).

In both the second and third interpretations, the layer ages derived from Equation (26) and used to form the age-depth relationship and $s(x, t)$ (Fig. 10 f and g) would be incorrect.

Here we favour the first interpretation for the following reasons. The second interpretation requires the accumulation rate to be coupled to the ice flow, most likely via interaction between atmospheric processes and surface topography. While such coupling is possible, for example via snow redistribution by katabatic wind (e.g. Black and Budd, 1964; King and others, 2004), we cannot envisage how it causes concerted changes where variations in the ice-flow velocity $u$ modulate the mean rate of accumulation and its anomalies by the same factor. The first interpretation needs a mechanism to fix $a(x)$ in space, and we find evidence for this and against the third interpretation. Figure 12 shows convincing correlation between the retrieved accumulation pattern $a(x)$ and the (along-flow) surface slope of the ice stream and also between the surface slope and the ice-stream bed topography. We interpret these correlations to mean that bed topographic undulations are inducing surface topography via ice-flow dynamics (this process is well recognized and studied in the literature; e.g. Gudmundsson, 2003); in turn, the surface topography creates anomalies in $a$, enhancing it on up-glacier-facing slopes and reducing it on down-glacierfacing slopes. Information given by Arcone and others (2005) for their figure 5, which shows a similar correlation between slope and accumulation on the ITASE traverse near the Core

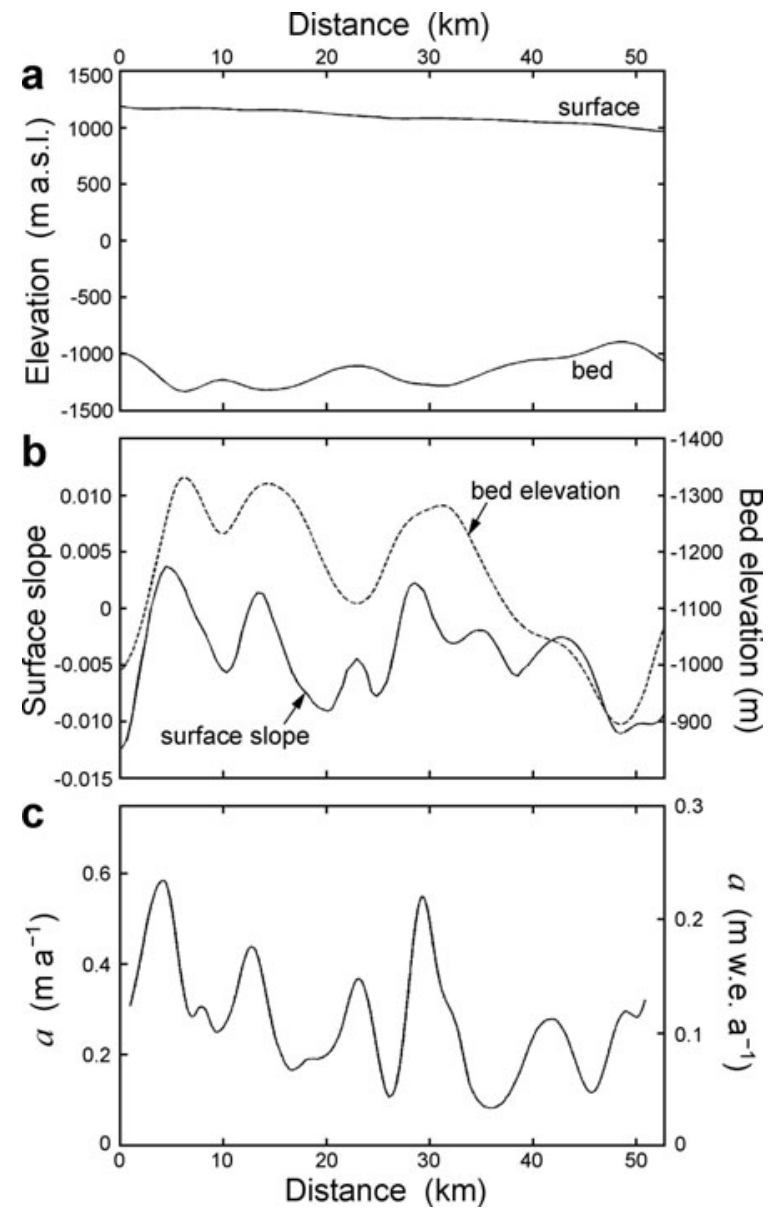

Fig. 12. Correlation between the retrieved mean accumulation-rate distribution $a(x)$ and the ice stream's cross-sectional geometry along the radar line. (a) Surface and bed-elevation profiles of the ice stream. (b) Surface slope in the ice-flow direction (solid curve) and the bed topography (dashed curve; plotted in inverted scale to highlight its relationship with surface slope). (c) The retrieved mean accumulation rate distribution $a(x)$ derived from Figure 10e. Comparison of (b) and (c) shows high accumulation rates on upglacier-facing slopes and low accumulation rates on down-glacierfacing slopes, if we define these slope types by using the mean slope as reference.

99-1 site (Fig. 1b), suggests that our up-facing and downfacing slopes are windward and leeward, respectively.

These mechanisms imply a basal origin and stationarity for the anomalies on $a(x)$ and flow stability in the onset zone. While this interpretation is specific to this case study and may not hold for other areas of the ice sheet, we note that the effect of basal topography on accumulation rates has been recognized independently by other radar studies (e.g. Rotschky and others, 2004). Furthermore, while the flowstability interpretation may not seem sensational, it adds to our knowledge of Bindschadler Ice Stream. One way to test it is to compare our layer-age estimates in Figure $10 \mathrm{f}$ with the layer-counted ages in a firn core drilled in our study area, or with the layer-counted ages in ITASE Core 99-1 after further radar profiling to link the isochrones between our study area and the core site.

\subsection{Hinge and slope migration}

We use the layer patterns in the synthetic and the real radargrams to evaluate the hinge and slope migration rates 

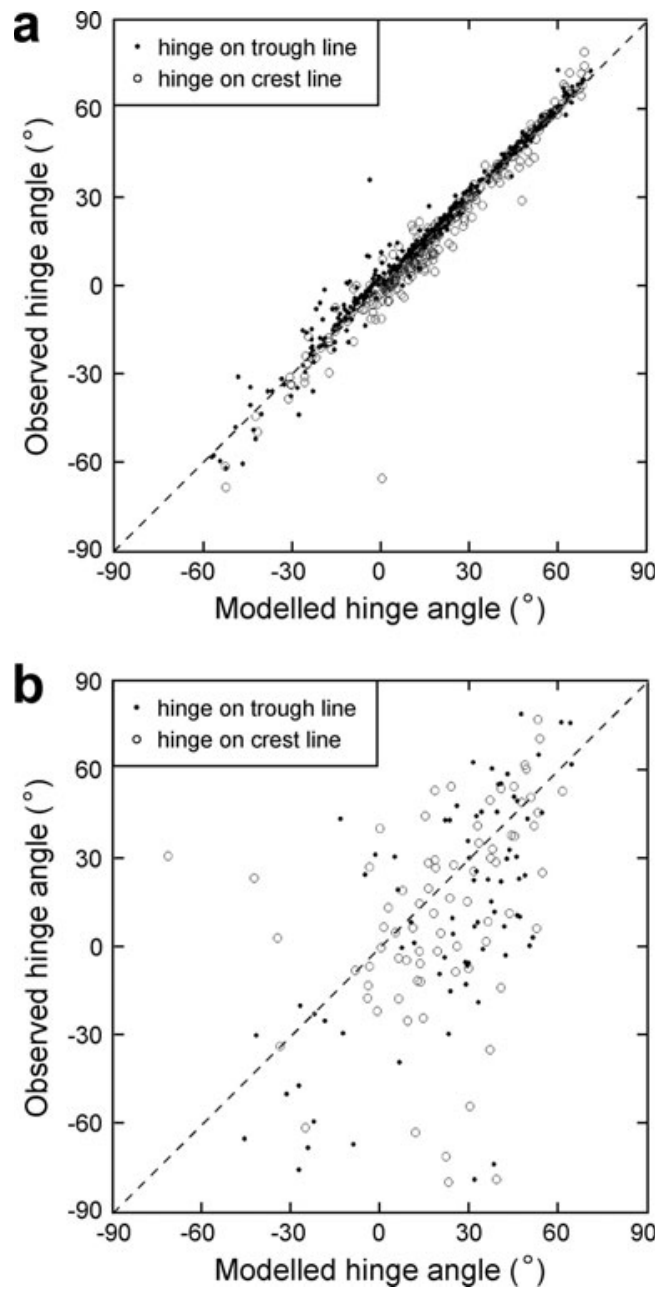

Fig. 13. Testing hinge-line Equation (23) with data from: (a) the synthetic radargram in Figure $6 \mathrm{~b}$ and (b) picked layers on the radargram used in our real case study. Vertical axes plot hinge-line angles measured from the transformed radargrams. Horizontal axes plot hinge-line angles predicted by Equation (23). See Section 3.4 for our definition of these angles.

predicted by theory, given by Equations (23) and (20), respectively:

$$
\begin{gathered}
\left.\frac{\mathrm{d} x}{\mathrm{~d} z}\right|_{s=0}=\frac{u_{0}-\frac{b(x)}{\partial s / \partial x}}{a(x)} \\
\left.\frac{\mathrm{d} x}{\mathrm{~d} t}\right|_{s=\text { const }}=v(x, t)=u_{0}-\frac{b(x)}{\partial s / \partial x} .
\end{gathered}
$$

Working in transformed coordinates, we tested each equation by a separate procedure. For each radargram we first compiled, at various $x$-positions, values of $a(x)$ and $b(x)$ as well as of $s$ and $\partial s / \partial x$ on its picked layers (the 61 layers of the synthetic radargram and the 17 picked layers in the real radargram). The quantity $u_{0}$ and the age difference between successive layers are known.

Equation (23) predicts the local dip of hinge lines, which have been traced on both radargrams (Figs $6 \mathrm{~b}$ and $10 \mathrm{~h}$ ). For each hinge-line segment between successive layers, we used the equation with the compiled data to calculate the values of $\mathrm{d} x / \mathrm{d} z$ at its two end points, then compared their mean with $\mathrm{d} x / \mathrm{d} z$ measured directly for the segment from the transformed radargram. We made this comparison for all traceable hinge-line segments, and defined their dip angles as $\tan ^{-1}(\mathrm{~d} x / \mathrm{d} z \div 100)$ (so that $0^{\circ}$ points downward) rather than measuring both up- and down-glacier dips against the horizon, which causes a sign discontinuity across the vertical. The factor of 100 here puts the angle more in line with the vertically exaggerated perspective of the radargrams shown in this paper.

Figure 13 plots the results, distinguishing those for crest and trough lines by different symbols. The strong correlation in Figure 13a for the synthetic radargram reveals the predictive power of Equation (23). By contrast, Figure 13b shows a weak, almost non-existent correlation for the real radargram. We attribute this to unsteadiness in its forcings (Section 3.3) and the large separations between picked layers, which introduce significant errors in the $\mathrm{d} x / \mathrm{d} z$ estimates in the comparison. We experimented with resampling the synthetic radargram at greater age interval and found that the scatter in Figure 13a is also caused by such errors, because it increased when we used fewer, more widely spaced isochrones.

Equation (20) predicts the migration velocity $v$ of a constant isochrone slope as we cross from one layer to a neighbouring (deeper) layer. In other words, starting from a given point on the first layer where its slope $s$ is known and using the age difference between the layers, this equation can be used to predict the 'target' point on the deeper layer where the same slope should occur. We tested Equation (20) by comparing the layer slope measured at the target point with its expected slope, for multiple positions on the layers on the real and synthetic radargrams. Figure $14 \mathrm{a}$ and $\mathrm{b}$ show the results. These plots exclude test positions where low isochrone curvature is expected to cause high migration velocities in Equation (20) and, together with the separation between layers, large prediction errors. Agreement between measured and expected slopes is strong for the synthetic radargram (Fig. 14a) and much weaker for the real radargram (Fig. 14b) (due again to the errors mentioned for the last test), but, unlike in Figure 13b, a noticeable correlation can be discerned here for the real radargram.

\section{CONCLUSIONS}

We have advanced a kinematic theory to explain the structure of layer undulations seen in flow-aligned radargrams of polar firn. It elucidates how spatially non-uniform accumulation and ice flow together shape isochrone geometry, and quantifies how layer folds encode these forcings when the latter do not vary in time. Unlike folding in rocks under shear or compression, which are typically caused by dynamical instabilities (Biot, 1957; Johnson and Fletcher, 1994), the mechanism here is kinematic because it results purely from differential velocities and does not involve internal forces. Analysis shows that isochrone slope propagates 'wave-like' across the radargram (and on an associated $x-t$ plane) so that hinge lines form loops, separating domains of rising and plunging isochrones. Predictions of this theory, including the rates of slope and hinge migration, find support in the radargram data from two case studies, which exemplify that the forcings behind complex layer patterns are not necessarily complex. By offering analytical insights on such patterns, the theory contributes a deeper understanding of a phenomenon whose study has relied on computational approaches.

In practical terms, we show that maps of isochrone slope for a given radargram carry decipherable information about 
its forcings (Figs $6 \mathrm{c}$ and $\mathrm{d}$ and $10 \mathrm{~g}$ and $\mathrm{h}$ ). We therefore advocate the compilation of these maps as a routine step in radargram analysis. With steady forcings, the arrangement of layer slopes on the $x-t$ plane obeys the scheme in Figure 7. One consequence of this is that the occurrence of coherent vertical zones of steeply plunging or steeply rising layers can be used to infer the sites of major accumulation peaks and troughs. A further consequence is that the age of isochrones and the accumulation rate distribution $a(x)$ can both be extracted from the radargram non-invasively, without needing firn cores (Section 3.2). This inversion yields $a(x) / u_{0}$ even if the ice-flow velocity $u_{0}$ is unknown and, despite its restrictive steady-flow assumption, can detect unsteadiness in the forcings.

Our application of this method to the onset zone of Bindschadler Ice Stream (Section 3.3) illustrates its potential for constraining the ice-flow history of a region on centurial timescales. While its use on many other areas of the ice sheets may not be possible currently due to the lack of flowaligned radargrams, suitable radargrams exist to enable comparison of several onset zones of streaming in West Antarctica. Such study can complement other investigations of the temporal variation of ice-stream systems and may yield more insights on the influence of basal topography on surface accumulation. In future studies, the data analysis could capitalize on a new automated method of extracting layer slopes from radargrams (Sime and others, 2011).

Firn densification is taken into account in our theory by the variable change in Equation (3). But, as we signalled in Section 2.1, a problem exists. Our starting layer-depth equation there, based on the densification descriptions of previous studies, is

$$
\rho(z) \frac{\partial z}{\partial t}+\frac{\partial}{\partial x}\left(u(x) \int_{0}^{z} \rho(\zeta) d \zeta\right)=\rho_{0} a(x)
$$

when written in the untransformed coordinates. The problem lies in the assumption of an invariant density-depth profile, $\rho(z)$, which overlooks the possibility that firn density can vary with horizontal distance and time as well as depth. Such outcome is in fact likely in the system we are studying, where firn compaction interacts with the accumulation and lateral advection. Even without the advection, the compaction rate depends on the local accumulation rate a (Herron and Langway, 1980; Arthern and others, 2010) so that spatial variations in a will cause $\rho$ to vary with $x$. Advection complicates this matter by putting each firn column under a time-varying accumulation rate, making the compaction history depend on material trajectories across the radar cross section.

These considerations imply two obstacles for models of isochrone evolution (whether used in the forward or inverse sense). First, the density becomes a spatio-temporally varying field $\rho(x, z, t)$ that must be found by solving a firn compaction model, coupled to Equation (28) via the vectorial velocity of the firn. This model requires testing against field measurements of firn density. The second obstacle is more subtle. Since the wave speed in firn depends on $\rho$ and $\rho$ varies with $x$ as well as $z$, when constructing radargrams from their time traces it may be inaccurate (even invalid) to assume a single depth-dependent profile of the wave speed for converting two-way travel time to depth. As Eisen and others (2008; p. 25) pointed out in their review, fieldworkers have probed this issue and found that although density-depth profiles can be spatially
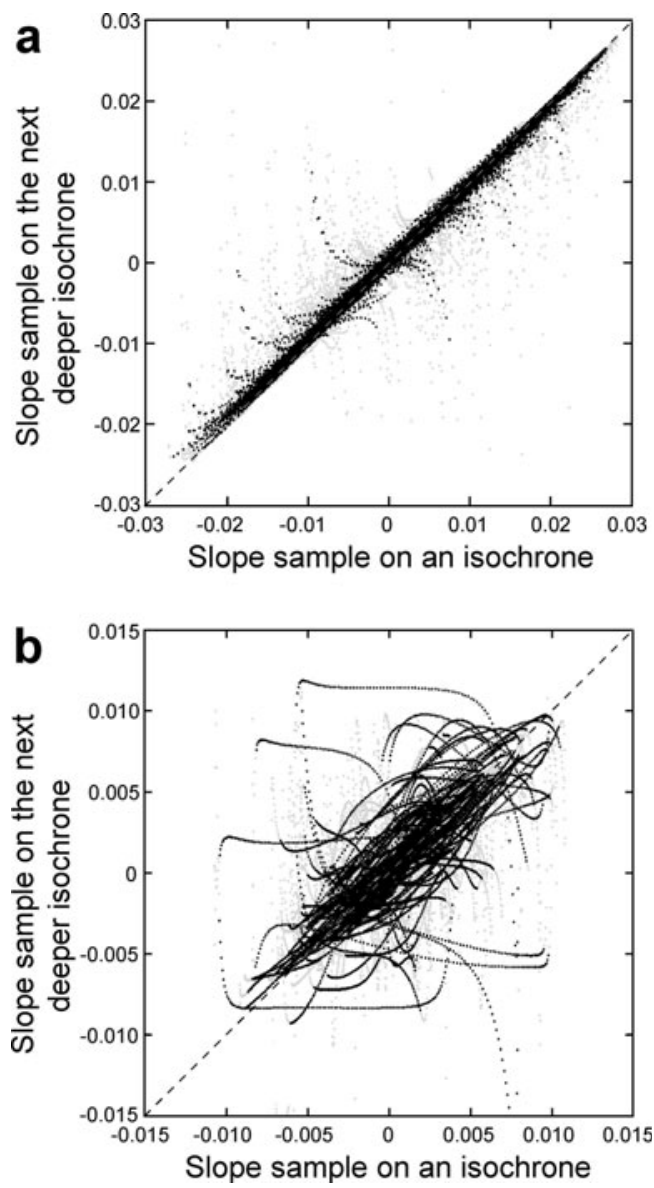

Fig. 14. Testing slope-migration Equation (20) with data from: (a) the synthetic radargram in Figure $6 \mathrm{~b}$ and (b) picked layers on the radargram used in our real case study. Horizontal axes plot isochrone slope $s$ measured on layers of the transformed radargrams. Vertical axes plot isochrone slope $s$ measured at positions on deeper layers adjacent to the original layers, positions predicted by Equation (20). In both panels, the grey points and black points, respectively, exclude test positions where absolute value of the isochrone curvature $|\partial s / \partial x|$ is $<5 \%$ and $<20 \%$ of the maximum $|\partial s / \partial x|$ found on the radargram.

homogeneous across plateau areas of the ice sheet, these profiles may show pronounced horizontal variations in areas with much accumulation variability and fast ice flow. In such areas, radargrams compiled with wave speeds measured (or interpolated) from a few isolated firn cores or common-midpoint surveys may contain serious errors. Strictly, the determination of layer depths then becomes entwined with our mathematical problem of the kinematic wave and compaction models: the radargram itself has to be found as part of the solution.

Besides modelling firn densification precisely, our theory could be extended to study the kinematic evolution of layer structures in 3-D (as revealed by radargrams with different alignments from the ice flow, intersecting each other) and to account for time-variable forcings. In the latter problem, we expect it to be much harder to gain mechanistic insights into fold geometries, as these will be governed by a slope surface that evolves with time. A different challenge is to address layer evolution across the firn-ice transition, i.e. to come up with a unified model that can link the undulations of our firn layers to those of englacial layers at depth where shearing is significant. 


\section{ACKNOWLEDGEMENTS}

We thank A. Morton for his hard work when gathering field data, and S. Arcone and O. Eisen for their useful reviews of our paper. The fieldwork yielding the radar and elevation data in Figures 1, 11 and 12 was supported by US National Science Foundation grant 86297 (to S. Anandakrishnan) and by the UK Natural Environmental Research Council (NERC) Geophysical Equipment Facility.

\section{REFERENCES}

Anschütz, H., D. Steinhage, O. Eisen, H. Oerter, M. Horwath and U. Ruth. 2008. Small-scale spatio-temporal characteristics of accumulation rates in western Dronning Maud Land, Antarctica. J. Glaciol., 54(185), 315-323.

Arcone, S.A., V.B. Spikes, G.S. Hamilton and P.A. Mayewski. 2004. Stratigraphic continuity in $400 \mathrm{MHz}$ short-pulse radar profiles of firn in West Antarctica. Ann. Glaciol., 39, 195-200.

Arcone, S.A., V.B. Spikes and G.S. Hamilton. 2005. Stratigraphic variation within polar firn caused by differential accumulation and ice flow: interpretation of a $400 \mathrm{MHz}$ short-pulse radar profile from West Antarctica. J. Glaciol., 51(174), 407-422.

Arthern, R.J., D.G. Vaughan, A.M. Rankin, R. Mulvaney and E.R. Thomas. 2010. In situ measurements of Antarctic snow compaction compared with predictions of models. J. Geophys. Res., 115(F3), F03011. (10.1029/2009JF001306.)

Bader, H. 1954. Sorge's Law of densification of snow on high polar glaciers. J. Glaciol., 2(15), 319-323.

Biot, M.A. 1957. Folding instability of a layered viscoelastic medium under compression. Proc. R. Soc. London, Ser. A, 242(1231), 444-454.

Black, H.P. and W. Budd. 1964. Accumulation in the region of Wilkes, Wilkes Land, Antarctica. J. Glaciol., 5(37), 3-15.

Carrier, G.F. and C.E. Pearson. 1988. Partial differential equations: theory and technique. Second edition. Boston, Academic Press.

Dixon, D., P.A. Mayewski, S. Kaspari, S. Sneed and M. Handley. 2004. A 200 year sub-annual record of sulfate in West Antarctica, from 16 ice cores. Ann. Glaciol., 39, 545-556.

Eisen, O. 2008. Inference of velocity pattern from isochronous layers in firn, using an inverse method. J. Glaciol., 54(187), 613-630.

Eisen, O. and 15 others. 2008. Ground-based measurements of spatial and temporal variability of snow accumulation in East Antarctica. Rev. Geophys., 46(RG2), RG2001. (10.1029/ 2006RG000218.)

Frezzotti, M., S. Urbini, M. Proposito, C. Scarchilli and S. Gandolfi. 2007. Spatial and temporal variability of surface mass balance near Talos Dome, East Antarctica. J. Geophys. Res., 112(F2), F02032. (10.1029/2006JF000638.)

Gray, L., E. King, H. Conway, B. Smith and F. Ng. 2005. Influence of ice dynamics in WAIS tributaries on firn layer stratigraphy. [Abstract] 12th West Antarctic Ice Sheet (WAIS) Workshop, 28 September-1 October, 2005, Sterling, VA. (http://www. waisworkshop.org/pastmeetings/abstracts05/Gray.pdf)

Gudmundsson, G.H. 2003. Transmission of basal variability to a glacier surface. J. Geophys. Res., 108(B5), 2253. (10.1029/ 2002JB002107.)

Herron, M.M. and C.C. Langway, Jr. 1980. Firn densification: an empirical model. J. Glaciol., 25(93), 373-385.

Hulbe, C. and M. Fahnestock. 2007. Century-scale discharge stagnation and reactivation of the Ross ice streams, West Antarctica. J. Geophys. Res., 112(F3), F03S27. (10.1029/ 2006JF000603.)

Johnson, A.M. and R.C. Fletcher. 1994. Folding of viscous layers: mechanical analysis and interpretation of structures in deformed rock. New York, Columbia University Press.
Joughin, I. and S. Tulaczyk. 2002. Positive mass balance of the Ross ice streams, West Antarctica. Science, 295(5554), 476-480.

King, E.C., D.L. Morse, R.B. Alley, S. Anandakrishnan, D.D. Blankenship and A.M. Smith. 2002. Radar profiles from the onset region of Ice Stream D1, Siple Coast, West Antarctica. [Abstract and Poster.] 9th West Antarctic Ice Sheet (WAIS) Workshop, 18-22 September 2002, Sterling, VA, USA. (http://www.waisworkshop. org/pastmeetings/abstracts02/King.html)

King, J.C., P.S. Anderson, D.G. Vaughan, G.W. Mann, S.D. Mobbs and S.B. Vosper. 2004. Wind-borne redistribution of snow across an Antarctic ice rise. J. Geophys. Res., 109(D11), D11104. (10.1029/2003JD004361.)

Koutnik, M.R. 2009. Inferring histories of accumulation rate, ice thickness, and ice flow from internal layers in glaciers and ice sheets. (PhD thesis, University of Washington.)

Nereson, N.A., C.F. Raymond, R.W. Jacobel and E.D. Waddington. 2000. The accumulation pattern across Siple Dome, West Antarctica, inferred from radar-detected internal layers. J. Glaciol., 46(152), 75-87.

$\mathrm{Ng}, \mathrm{F}$. and H. Conway. 2004. Fast-flow signature in the stagnated Kamb Ice Stream, West Antarctica. Geology, 32(6), 481-484.

Parrenin, F. and R. Hindmarsh. 2007. Influence of a non-uniform velocity field on isochrone geometry along a steady flowline of an ice sheet. J. Glaciol., 53(183), 612-622.

Press, W.H., S.A. Teukolsky, W.T. Vetterling and B.P. Flannery. 1992. Numerical recipes in C: the art of scientific computing. Second edition. Cambridge, Cambridge University Press.

Price, S.F., R.A. Bindschadler, C.L. Hulbe and D.D. Blankenship. 2002. Force balance along an inland tributary and onset to Ice Stream D, West Antarctica. J. Glaciol., 48(160), 20-30.

Rotschky, G., O. Eisen, F. Wilhelms, U. Nixdorf and H. Oerter. 2004. Spatial distribution of surface mass balance on Amundsenisen plateau, Antarctica, derived from ice-penetrating radar studies. Ann. Glaciol., 39, 265-270.

Siegert, M.J., A.J. Payne and I. Joughin. 2003. Spatial stability of Ice Stream $D$ and its tributaries, West Antarctica, revealed by radio-echo sounding and interferometry. Ann. Glaciol., 37, 377-382.

Siegert, M.J. and 9 others. 2004. Ice flow direction change in Interior West Antarctica. Science, 305(5692), 1948-1951.

Sime, L.C., R.C.A. Hindmarsh and H.F.J. Corr. 2011. Automated processing to derive dip angles of englacial radar reflectors in ice sheets. J. Glaciol., 57(202), 260-266.

Spikes, V.B., G.S. Hamilton, S.A. Arcone, S. Kaspari and P. Mayewski. 2004. Variability in accumulation rates from GPR profiling on the West Antarctic plateau. Ann. Glaciol., 39, 238-244.

Steig, E.J. and 16 others. 2005. High-resolution ice cores from US ITASE (West Antarctica): development and validation of chronologies and determination of precision and accuracy. Ann. Glaciol., 41, 77-84.

Vaughan, D.G., H.F.J. Corr, C.S.M. Doake and E.D. Waddington. 1999. Distortion of isochronous layers in ice revealed by ground-penetrating radar. Nature, 398(6725), 323-326.

Vaughan, D.G., P.S. Anderson, J.C. King, G.W. Mann, S.D. Mobbs and R.S. Ladkin. 2004. Imaging of firn isochrones across an Antarctic ice rise and implications for patterns of snow accumulation rate. J. Glaciol., 50(170), 413-418.

Waddington, E.D., T.A. Neumann, M.R. Koutnik, H.-P. Marshall and D.L. Morse. 2007. Inference of accumulation-rate patterns from deep layers in glaciers and ice sheets. J. Glaciol., 53(183), 694-712.

Whitham, G.B. 1974. Linear and non-linear waves. New York, etc., John Wiley and Sons.

Woodward, J. and E.C. King. 2009. Radar surveys of the Rutford Ice Stream onset zone, West Antarctica: indications of flow (in)stability? Ann. Glaciol., 50(51), 57-62. 